\title{
Heat flows for extremal Kähler metrics
}

\author{
SAntiago R. SimanCA
}

\begin{abstract}
Let $(M, J, \Omega)$ be a closed polarized complex manifold of Kähler type. Let $G$ be the maximal compact subgroup of the automorphism group of $(M, J)$. On the space of Kähler metrics that are invariant under $G$ and represent the cohomology class $\Omega$, we define a flow equation whose critical points are the extremal metrics, i.e. those that minimize the square of the $L^{2}$-norm of the scalar curvature. We prove that the dynamical system in this space of metrics defined by the said flow does not have periodic orbits, and that its only fixed points are the extremal metrics. We prove local time-existence of the flow, and conclude that if the lifespan of the solution is finite, then the supremum of the norm of its curvature tensor must blow up as time approaches it. While doing this, we also prove that extremal solitons can only exist in the non-compact case, and that the range of the holomorphy potential of the scalar curvature is an interval independent of the metric chosen to represent $\Omega$. We end up with some conjectures concerning the plausible existence and convergence of global solutions under suitable geometric conditions.
\end{abstract}

Mathematics Subject Classification (2000): 53C55 (primary); 35K55, 58E11, 58J35 (secondary).

\section{Introduction}

We define and study a new dynamical system in the space of Kähler metrics that represent a fixed cohomology class on a given closed complex manifold of Kähler type. The critical points of this flow are extremal metrics, that is to say, minimizers of the functional defined by the $L^{2}$-norm of the scalar curvature. We derive the equation, describe some of its general properties, and prove that, given an initial data, the equation has a unique classical solution on some time interval. It would be of great interest to know if the solution exists for all times, or whether it develops some singularities in finite time. We have no general answer to this yet. However, we show some evidence indicating that, in some specific cases, the solution should exist for all time and converge to an extremal metric as time goes to infinity.

In order to put our equation in proper perspective, we begin by recalling a different but related one, the Ricci flow. Let $M$ be a compact manifold $M$ of dimension $n$. Given a metric $g$, we denote its Ricci tensor by Ricci $g$ and its average scalar 
curvature by $r_{g}$. The Ricci flow

$$
\frac{d g}{d t}=2\left(\frac{r_{g}}{n} g-\operatorname{Ricci}_{g}\right),
$$

was introduced by R. Hamilton [11] as a mechanism to improve the properties of its initial data. It is a non-linear heat equation in the metric, which hopefully becomes better as time passes by in the same way as the heat equation improves an initial distribution of heat in a given region, and makes it uniform all throughout as time goes to infinity. Hamilton used it to show that on a three dimensional manifold, an initial metric of positive Ricci curvature flows according to this equation towards a limit that has constant positive sectional curvature.

In the case of a Kähler manifold, Hamilton's flow equation may be used when seeking a Kähler-Einstein metric on the said manifold. Of course, this would a priori require that the first Chern class $c_{1}$ be signed, so that it may be represented by Kähler-Einstein metrics, or their opposites. Regardless of that consideration, the idea inspired Cao [5] to study the equation

$$
\frac{d \omega}{d t}=\eta-\rho_{t},
$$

for $\eta$ a fixed real closed $(1,1)$-form representing the class $c_{1}(M)$. Here, $\omega$ and $\rho$ are the Kähler and Ricci forms of the metric, respectively. Using Yau's work on the Calabi conjecture, he proved that solutions exists for all $t \geq 0$ and that the path of metrics so defined converges to a Kähler metric with the prescribed Ricci form $\eta$ as $t \rightarrow \infty$. He went on and, under the assumption that $c_{1}(M)<0$, replaced $\eta$ in the equation above by $-\omega_{t}$ and proved that the corresponding solution to the initial value problem exists for all times and converges to a Kähler-Einstein metric as $t \rightarrow \infty$, rederiving the now famous version of the theorem of Yau and Aubin.

As good as the Ricci flow may be, when it converges it can only do so to an Einstein metric, which is only possible under a priori conditions on $M$ that might not hold in general. We are interested in studying polarized Kähler manifolds, and in finding canonical representatives of the polarization. Our flow is adapted to accomplishing this other somewhat different goal.

If $(M, J, \Omega)$ is a polarized Kähler manifold, for a variety of technical reasons to be clarified below, we consider metrics that are invariant under the action of a maximal compact subgroup of the automorphism group of $(M, J)$, and introduce the equation

$$
\frac{d \omega}{d t}=\Pi_{t} \rho-\rho_{t},
$$

with initial condition a given metric representing $\Omega$. Here $\Pi_{t}$ is a metric-dependent projection operator that intertwines the metric trace with the $L^{2}$-orthogonal projection $\pi_{t}$ onto the space of real holomorphy potentials, these being those real-valued functions whose gradients are holomorphic vector fields. The projection $\Pi_{t}$ is such that $\Pi_{t} \rho_{t}-\rho_{t}$ is cohomologous to zero, and so all metrics satisfying the equation represent $\Omega$. As such, the critical points of the equation, i.e. the metrics for which $\Pi \rho=\rho$, are precisely those metrics whose scalar curvatures have holomorphic gradients namely the extremal metrics of Calabi [3]. This fact constitutes the guiding principle behind our consideration of this new flow equation. 
In general, our flow equation is different from the Kähler version of the Ricci flow, even when $\Omega= \pm c_{1}$. This last assertion is illustrated, for instance, by the blow up of $\mathbb{C P}^{2}$ at one or two points, and the reason is basically a simple one: extremal metrics, which is what we seek when we consider the new flow, is a concept that imposes milder conditions than those required for the metric to be Kähler-Einstein, and when $\Omega=c_{1}$, the two concepts agree only if we know additional restrictions on $c_{1}[7,20]$. These do not hold on the two mentioned examples. On the other hand, the flows always coincide on Riemann surfaces (where they also agree with the two-dimensional version of the Yamabe flow [10]) because, regardless of the metric $g$ under consideration, the holomorphy potential $\pi_{g} s_{g}$, where $s_{g}$ is the scalar curvature of $g$, is a topological constant determined by the Euler characteristic. In these cases, it is still of some interest to point out that the extremal flow always arises as the gradient flow of a Riemannian functional, statement that in this dimension cannot be ascertained for the other two flows in light of the Gauss-Bonnet theorem.

The main point of the present article will be to show that solutions to the extremal flow equation exists locally in time. However, even if these were going to exist globally, we should not expect that they would converge as time approaches infinity in all possible cases. We already know examples of Kählerian manifolds that do not admit extremal metrics [2, 16, 22]. We also have already a partial picture that explains why these examples exist. Those in [16] fail to satisfy a necessary condition on the space of holomorphic vector fields, while those in [2] and [22] are related to stability of the manifold under deformations of the complex structure, property that is independent of and not reflected by those of the Lie algebra of holomorphic vector fields.

Pointing more to the heart of the problem that interests us here, we had proven [15] that the set of Kähler classes that can be represented by extremal metrics is open in the Kähler cone. The study of the extremal flow equation above, and its potential convergence to a limit extremal metric, can be seen as a general method that could decide if the the extremal cone is - or is not-closed too.

In proving local time existence of the extremal flow, we also show that if the lifespan is finite, then the point wise norm of the curvature tensor must blow up as times approaches it. We leave for later the analysis of global existence and convergence under suitable geometric conditions, in particular, the analysis of these issues for surfaces with positive first Chern class, where our flow equation seems to be a promising tool for the resolution of the extremal metric problem.

We organize the paper as follows: in Section 2 we recall the notion and basic facts about extremal metrics; in Section 3 we explain in detail the derivation of the extremal flow equation, and prove general results about it; in Section 4 we linearize this flow equation, showing that it results into a pseudo-differential perturbation of the standard time-dependent heat equation. This form of the linearization is an essential fact in our proof of local time existence, which is done in Section 5 via a fixed point-type of argument. We end with some remarks justifying our hope that solutions to the extremal flow will converge to an extremal metric under suitable general geometric conditions. 


\section{Extremal Kähler Metrics}

Let $(M, J, g)$ be a closed Kähler manifold of complex dimension $n$. This means that $(M, J)$ is a closed complex manifold and that $\omega(X, Y):=g(J X, Y)$, which is skew-symmetric because $g$ is a Hermitian Riemannian metric, is a closed 2-form. The form $\omega$ is called the Kähler form, and its cohomology class $[\omega] \in H^{2}(M, \mathbb{R})$ is called the Kähler class.

We denote by $\mathfrak{h}=\mathfrak{h}(M, J)$ the complex Lie algebra of holomorphic vector fields of $(M, J)$. Since $M$ is compact, this is precisely the Lie algebra of the group of biholomorphism of $(M, J)$. The subset $\mathfrak{h}_{0}$ of holomorphic vector fields with zeroes is an ideal of $\mathfrak{h}$, and the quotient algebra $\mathfrak{h} / \mathfrak{h}_{0}$ is Abelian.

We shall say that $(M, J)$ is a generic complex manifold of Kähler type if $\mathfrak{h}_{0}$ is trivial. Typically, complex manifolds carry no non-zero holomorphic vector fields and are, therefore, generic in our sense. However, our definition includes also those complex manifolds whose non-zero holomorphic fields have empty zero sets.

By complex multi-linearity, we may extend the metric $g$, the Levi-Civita connection $\nabla$ and the curvature tensor $R$ to the complexified tangent bundle $\mathbb{C} \otimes T M$. Since $\mathbb{C} \otimes T M$ decomposes into the $\pm i$-eigenspaces of $J, \mathbb{C} \otimes T M=T^{1,0} M \oplus$ $T^{0,1} M$, we can express any tensor field or differential operator in terms of the corresponding decomposition. For example, if $\left\{z^{1}, \ldots, z^{n}\right\}$ is a holomorphic coordinate system on $M$, we get induced bases $\left\{\partial_{z^{\jmath}}\right\}$ and $\left\{\partial_{z^{j}}:=\partial_{\bar{z}^{\prime}}\right\}$ for $T^{1,0} M$ and $T^{0,1} M$, respectively, and if we express the metric $g$ in terms of this basis by setting $g_{\mu v}:=g\left(\partial_{z^{\mu}}, \partial_{z^{v}}\right)$, where the indices $\mu, v$ range over $\{1, \ldots, n, \overline{1}, \ldots, \bar{n}\}$, it follows from the condition that $g$ be Hermitian that $g_{J k}=g_{\bar{\jmath} \bar{k}}=0$, and that $\omega=\omega_{j \bar{k}} d z^{j} \wedge d \bar{z}^{k}=i g_{j} d z^{J} \wedge d \bar{z}^{k}$.

The complexification of the exterior algebra can be decomposed into a direct sum of of forms of type $(p, q)$. Indeed, we have $\wedge^{r} M=\bigoplus_{p+q=r} \wedge^{p, q} M$. The integrability of $J$ implies that the exterior derivative $d$ splits as $d=\partial+\bar{\partial}$, where $\partial: \wedge^{p, q} M \rightarrow \wedge^{p+1, q} M, \bar{\partial}: \wedge^{p, q} M \rightarrow \wedge^{p, q+1} M, \partial^{2}=\bar{\partial}^{2}=0$ and $\partial \bar{\partial}=-\bar{\partial} \partial$. Complex conjugation also extends, and we define a form to be real if it is invariant under this operation. An important result in Kähler geometry is that, given a $d$-exact real form $\beta$ of type $(p, p)$, there exists a real form $\alpha$ of type $(p-1, p-1)$ such that $\beta=i \partial \bar{\partial} \alpha$.

The Ricci form $\rho$ is defined in terms of the Ricci tensor $r$ of $g$ by $\rho(X, Y)=$ $r(J X, Y)$. It is a closed form whose components are given by

$$
r_{j \bar{k}}=-i \rho_{j \bar{k}}=-\frac{\partial^{2}}{\partial z^{j} \partial \bar{z}^{k}} \log \operatorname{det}\left(g_{p \bar{q}}\right) .
$$

The form $\frac{1}{2 \pi} \rho$ is the curvature of the canonical line bundle $\kappa=\Lambda^{n}\left(T^{*} M\right)^{1,0}$, and represents the first Chern class $c_{1}=c_{1}(M, J)$.

The scalar curvature $s$ is, by definition, the trace $s=r_{\mu}^{\mu}=2 g^{j}{ }^{\bar{k}} r_{j \bar{k}}$ of the Ricci tensor, and can be conveniently calculated by the formula

$$
s \omega^{\wedge n}=2 n \rho \wedge \omega^{\wedge(n-1)} .
$$


Since the volume form is given by $d \mu=\frac{\omega^{\wedge} n}{n !}$ and $M$ is compact, this formula implies that

$$
\int_{M} s d \mu=\frac{4 \pi}{(n-1) !} c_{1} \cup[\omega]^{\cup(n-1)},
$$

a quantity that only depends upon the complex structure $J$ and the cohomology class $[\omega]$, and that generalizes the well-known Gauss-Bonnet theorem for surfaces. Notice that $\int_{M} d \mu=\frac{1}{n !}[\omega]^{\cup n}$, and so the average scalar curvature

$$
s_{0}=4 \pi n \frac{c_{1} \cup[\omega]^{n-1}}{[\omega]^{n}},
$$

is also a quantity that depends only on the Kähler class $[\omega]$ and the homotopy class of the complex-structure tensor $J$.

Suppose that $(M, J)$ is polarized by a positive class $\Omega \in H^{1,1}(M, \mathbb{C}) \cap$ $H^{2}(M, \mathbb{R})$. Let $\mathfrak{M}_{\Omega}$ be the set of all Kähler forms representing $\Omega$. Since any two elements $\tilde{\omega}$ and $\omega$ of $\mathfrak{M}_{\Omega}$ are such that $\tilde{\omega}=\omega+i \partial \bar{\partial} \varphi$ for some real-valued potential function $\varphi$, at the expense of fixing a background Kähler metric $\omega$ that represents $\Omega$, we can describe $\mathfrak{M}_{\Omega}$ as $\mathfrak{M}_{\Omega}=\left\{\omega_{\varphi}=\omega+i \partial \bar{\partial} \varphi: \omega_{\varphi}>0\right\}$. Thus, $\mathfrak{M}_{\Omega}$ is an affine space modeled on an open subset of the space of smooth functions that parameterizes the deformations of the base point $\omega$. We may topologize it by defining a suitable topology on the space of deformation potentials.

In what follows, we shall not distinguish between the Kähler metric and its Kähler form, passing from one to the other at will.

Consider the functional

$$
\begin{aligned}
\mathfrak{M}_{\Omega} & \stackrel{\Phi_{\Omega}}{\longrightarrow} \mathbb{R} \\
\omega & \mapsto \int_{M} s_{\omega}^{2} d \mu_{\omega},
\end{aligned}
$$

where the metric associated with the form $\omega$ has scalar curvature $s_{\omega}$ and volume form $d \mu_{\omega}$. A critical point of this functional is by definition an extremal Kähler metric [3]. These metrics were introduced by E. Calabi with the idea of seeking canonical representatives of $\Omega$.

Given any Kähler metric $g$, a smooth complex-valued function $f$ gives rise to the $(1,0)$ vector field $f \mapsto \partial^{\#} f=\partial_{g}^{\#} f$ defined by the expression

$$
g\left(\partial^{\#} f, \cdot\right)=\bar{\partial} f .
$$

This vector field is holomorphic if and only if we require that $\bar{\partial} \partial^{\#} f=0$, condition equivalent to $f$ being in the kernel of the operator

$$
L_{g} f:=\left(\bar{\partial} \partial^{\#}\right)^{*} \bar{\partial} \partial^{\#} f=\frac{1}{4} \Delta^{2} f+\frac{1}{2} r^{\mu \nu} \nabla_{\mu} \nabla_{\nu} f+\frac{1}{2}\left(\nabla^{\bar{\ell}} \sigma\right) \nabla_{\bar{\ell}} f .
$$


The ideal $\mathfrak{h}_{0}$ consists of vector fields of the form $\partial_{g}^{\#} f$, for a function $f$ in the kernel of $L_{g}$. In other words, a holomorphic vector field $\Xi$ can be written as $\partial_{g}^{\#} f$ if and only if the zero set of $\Xi$ is non-empty.

The variation of $\Phi_{\Omega}$ can be given in terms of the operator $L_{g}$ above. Indeed,

$$
\left.\frac{d}{d t} \Phi_{\Omega}(\omega+t i \partial \bar{\partial} \varphi)\right|_{t=0}=-4 \int s_{\omega} L_{\omega} \varphi d \mu_{\omega}
$$

Hence, the Euler-Lagrange equation for a critical point $g$ of (2.4) can be cast as the fact that the scalar curvature $s_{g}$ is a real-valued function in the kernel of $L_{g}$. Thus, the vector field $\partial_{g}^{\#} s_{g}$ must be holomorphic and its imaginary part is a Killing field of $g$.

Given a Kähler metric $\omega$, its normalized Ricci potential $\psi_{\omega}$ is defined to be the only function orthogonal to the constants such that $\rho=\rho_{H}+i \partial \bar{\partial} \psi_{\omega}$, where $\rho_{H}$ is the $\omega$-harmonic component of $\rho$. In terms of the scalar curvature and its projection onto the constants, we have that $\psi_{\omega}=-G_{\omega}\left(s_{\omega}-s_{0}\right)$. If $\mathcal{K}$ denotes the Kähler cone of $(M, J)$, the Futaki character [7] is defined to be the map

$$
\begin{aligned}
& \mathfrak{F}: \mathfrak{h} \times \mathcal{K} \longrightarrow \mathbb{C} \\
& \mathfrak{F}(\Xi,[\omega])=2 \int_{M} \Xi\left(\psi_{\omega}\right) d \mu=-2 \int_{M} \Xi\left(G_{\omega}\left(s_{\omega}-s_{0}\right)\right) d \mu_{\omega} .
\end{aligned}
$$

It is calculated using a particular representative $\omega$ of the class, but it depends only on $\Omega$ and not on the particular choice of representative [7, 4]. And when applied to a holomorphic vector field of the form $\Xi=\partial^{\#} f$, it yields

$$
\mathfrak{F}(\Xi,[\omega])=-\int_{M} f\left(s_{\omega}-s_{0}\right) d \mu_{\omega} .
$$

If a metric $\omega \in \mathfrak{M}_{\Omega}$ is extremal, we may apply (2.7) to the vector field $\partial_{\omega}^{\#} s_{\omega}$ and obtain that $\mathfrak{F}\left(\partial_{\omega}^{\#} s_{\omega}, \Omega\right)=-\left\|s_{\omega}-s_{0}\right\|^{2}$. Thus, the Futaki character represents an obstruction to $\omega$ being a metric of constant scalar curvature.

Extremal metrics achieve the infimum of $\Phi_{\Omega}$ over $\mathfrak{M}_{\Omega}$. In fact, the critical value $E(\Omega)$ they achieve is a differentiable function of $\Omega$ [19], and there exists a holomorphic vector field $X_{\Omega}[8]$ such that

$$
\Phi_{\Omega}(\omega) \geq E(\Omega):=s_{0}^{2} \frac{\Omega^{n}}{n !}-\mathfrak{F}\left(X_{\Omega}, \Omega\right)
$$

for all $\omega \in \mathfrak{M}_{\Omega}$.

\section{Derivation of the evolution equation}

Calabi [4] showed that the identity component of the isometry group of an extremal Kähler metric $g$ is a maximal compact subgroup of the identity component of the biholomorphism group of $(M, J)$. This implies that, up to conjugation, the 
identity components of the isometry groups of extremal Kähler metrics coincide [15]. Therefore, modulo biholomorphisms, the search for extremal Kähler metrics is completely equivalent to the search for extremal metrics among those that are invariant under the action of a fixed maximal compact subgroup of the connected biholomorphism group. This last problem, however, turns out to be technically easier to analyze.

\subsection{Real holomorphy potentials}

Given any Kähler metric $g$ on $(M, J)$, every complex-valued function $f$ in the kernel of $L_{g}=\left(\bar{\partial} \partial_{g}^{\#}\right)^{*} \bar{\partial} \partial_{g}^{\#}$ in (2.5) is associated with the holomorphic vector field $\Xi=\partial_{g}^{\#} f$, and since the operator $L_{g}$ is elliptic, the space of such functions is finitedimensional. However, $L_{g}$ is not, generally speaking, a real operator. Therefore, the real and imaginary parts of a function in its kernel do not have to be elements of the kernel also. It has been proven elsewhere [15] that if $f$ is a real-valued function in the kernel of $L_{g}$, then the imaginary part of $\partial_{g}^{\#} f$ is a Killing field of $g$, and that a Killing field arises in this way if, and only if, its zero set is not empty.

Let $G$ be a maximal compact subgroup of the biholomorphism group of $(M, J)$, and $g$ be a Kähler metric on $M$ with Kähler class $\Omega$. Without loss of generality, we assume that $g$ is $G$-invariant. We denote by $L_{k, G}^{2}$ the real Hilbert space of $G$ invariant real-valued functions of class $L_{k}^{2}$, and consider $G$-invariant deformations of this metric preserving the Kähler class:

$$
\tilde{\omega}=\omega+i \partial \bar{\partial} \varphi, \quad \varphi \in L_{k+4, G}^{2}, k>n .
$$

In this expression, the condition $k>n$ ensures that $L_{k, G}^{2}$ is a Banach algebra, making the scalar curvature of $\tilde{\omega}$ a well-defined function in the space.

We denote by $\mathfrak{g} \subset \mathfrak{h}$ the Lie algebra of $G$, and by $\mathfrak{z}$ the center of $\mathfrak{g}$. We let $\mathfrak{z}_{0}=\mathfrak{z} \cap \mathfrak{g}_{0}$, where $\mathfrak{g}_{0} \subset \mathfrak{g}$ is the ideal of Killing fields which have zeroes. If $\tilde{g}$ is any $G$-invariant Kähler metric on $(M, J)$, then each element of $\mathfrak{z}_{0}$ is of the form $J \nabla_{\tilde{g}} f$ for a real-valued solution of (2.5). In fact, $\mathfrak{z}_{0}$ corresponds to the set of real solutions $f$ which are invariant under $G$, since

$$
\partial^{\#}: \operatorname{ker}\left[\left(\bar{\partial} \partial_{\tilde{g}}^{\#}\right)^{*} \bar{\partial} \partial_{\tilde{g}}\right] \rightarrow \mathfrak{h}_{0}
$$

is a homomorphism of $G$-modules.

The restriction of $\operatorname{ker}\left(\bar{\partial} \partial_{\tilde{g}}^{\#}\right) * \bar{\partial} \partial_{\tilde{g}}$ to $L_{k+4, G}^{2}$ depends smoothly on the $G$-invariant metric $\tilde{g}$. Indeed, choose a basis $\left\{X_{1}, \ldots, X_{m}\right\}$ for $\mathfrak{z}_{0}$, and, for each $(1,1)$-form $\chi$ on $(M, J)$, consider the set of functions

$$
\begin{aligned}
& p_{0}(\chi)=1 \\
& p_{j}(\chi)=2 i G_{g} \bar{\partial}_{g}^{*}\left(\left(J X_{j}+i X_{j}\right) \downarrow \chi\right), \quad j=1, \ldots, m
\end{aligned}
$$

where $G_{g}$ is the Green operator of the metric $g$. If $\tilde{\omega}$ is the Kähler form of the $G$ invariant metric $\tilde{g}$, then $\partial_{\tilde{g}}^{\#} p_{j}(\tilde{\omega})=J X_{j}+i X_{J}$, and the set $\left\{p_{j}(\tilde{\omega})\right\}_{j=0}^{m}$ consists of 
real-valued functions and forms a basis for $\operatorname{ker}\left(\bar{\partial} \partial_{\tilde{g}}^{\#}\right){ }^{*} \bar{\partial} \partial_{\tilde{g}}$. Furthermore, for metrics $\tilde{\omega}$ as in (3.1), the map $\varphi \mapsto p_{j}(\omega+i \partial \bar{\partial} \varphi)$ is, for each $j$, bounded as a linear map from $L_{k+4, G}^{2}$ to $L_{k+3, G}^{2}$.

With respect to the fixed $L^{2}$ inner product, let $\left\{f_{\tilde{\omega}}^{0}, \ldots, f_{\tilde{\omega}}^{m}\right\}$ be the orthonormal set extracted from $\left\{p_{j}(\tilde{\omega})\right\}$ by the Gram-Schmidt procedure. We then let

$$
\begin{aligned}
\pi_{\tilde{\omega}}: L_{k, G}^{2} & \rightarrow L_{k, G}^{2} \\
u & \mapsto \sum_{j=0}^{m}\left\langle f_{\tilde{\omega}}^{j}, u\right\rangle_{L^{2}} f_{\tilde{\omega}}^{j}
\end{aligned}
$$

denote the associated projector. In fact, by the regularity of the functions $\left\{p_{1}, \ldots, p_{m}\right\}$, this projection can be defined on $L_{k+j, G}^{2}$ for $j=0,1,2,3$, and for metrics as in (3.1), the map $\varphi \mapsto \pi_{\tilde{\omega}}$ is smooth from a suitable neighborhood of the origin in $L_{k+4, G}^{2}$ to the real Hilbert space $\operatorname{End}\left(L_{k+j, G}^{2}\right) \cong \bigotimes^{2} L_{k+j, G}^{2}$.

The holomorphic vector field $X_{\Omega}$ of the class that yields the lower bound (2.8) is given by $X_{\Omega}=\partial_{g}^{\#}\left(\pi_{g} s_{g}-s_{0}\right)$. As such, it may depend on the choice of maximal compact subgroup $G$ of the automorphism group of $(M, J)$, but the value of $\mathfrak{F}\left(X_{\Omega}, \Omega\right)$ does not. The critical value $E(\Omega)$, or energy of the class $\Omega$, is nothing more than

$$
E(\Omega)=\int\left(\pi_{g} s_{g}\right)^{2} d \mu_{g} .
$$

(This way of computing the energy of the class through $G$-invariant metrics is very convenient and has been used several times elsewhere [18, 20,21] with other (but related) purposes in mind.)

Remark 3.1. For generic manifolds $(M, J)$, the space of real holomorphy potentials consists of the constant functions only. Thus, $\pi_{g} s_{g}$ is the constant given in (2.3) no matter what $G$-invariant representative $g$ of the class $\Omega$ we consider. However, this last function could be constant even under the presence of non-trivial holomorphic vector fields with zeroes. For that, the Futaki invariant of the class must vanish, and in that case, the vector field $X_{\Omega}$ associated to $\Omega$ is trivial.

A simple instance of this is given by a compact Riemann surface $\Sigma$, where for any $G$-invariant metric $g$, we have that $\pi_{g} s_{g}=4 \pi \chi(\Sigma) / \mu_{g}(\Sigma)$ where $\chi(\Sigma)$ is the Euler characteristic. Indeed, Riemann surfaces are either hyperbolic, parabolic or elliptic. The first two of these are generic, and the assertion is clear then. For the remaining case, that of the Riemann sphere, the space $\mathfrak{h}_{0}$ is non-trivial but the Futaki character vanishes. The assertion follows from the Gauss-Bonnet theorem embodied in (2.2), and the identity (2.7) applied to $f=\pi_{g} s_{g}$.

From now on, we shall denote by $\mathfrak{M}_{\Omega, G}$ the set of $G$-invariant Kähler metrics representing the class $\Omega$. Given a path of metrics $\omega_{t} \in \mathfrak{M}_{\Omega, G}$, since the kernel of $\left(\bar{\partial} \partial_{\tilde{g}}^{\#}\right)^{*} \bar{\partial} \partial_{\tilde{g}}$ depends smoothly on $\tilde{g}$, the differential of $\pi_{t} s_{t}$ is well-defined. Here, $\pi_{t}$ and $s_{t}$ are the projection operator and scalar curvature associated to the metric $\omega_{t}$, respectively. Since $\pi_{t} s_{t}$ is of order four in the potential of the metric, naively we would expect its differential to be an operator of order four on the tangent space to 
$\mathfrak{M}_{\Omega, G}$ at $\omega_{t}$. However, we get something significantly better, and gain quite a bit of regularity. This fact will be very convenient later on.

Lemma 3.2. Let $\omega_{t}=\omega+i \partial \bar{\partial} \varphi_{t}$ be a path of metrics in $\mathfrak{M}_{\Omega, G}$ with $\omega_{0}=\omega$. Consider the projection $\pi_{t} s_{t}$ of the scalar curvature $s_{t}$ onto the space of real holomorphy potentials, and let $\dot{\varphi}_{t}=\frac{d}{d t} \varphi_{t}$. Then

$$
\left.\frac{d}{d t}\left(\pi_{t} s_{t}\right)=\partial \dot{\varphi}_{t}\right\lrcorner X_{\Omega}=\left(\partial^{\#} \dot{\varphi}_{t}, X_{\Omega}\right)_{t}=\left(\partial \dot{\varphi}_{t}, \partial\left(\pi_{t} s_{t}\right)\right)_{t},
$$

where $X_{\Omega}=\partial_{t}^{\#} \pi_{t} s_{t}$ is the holomorphic vector field of the class $\Omega$. In particular, this derivative is a differential operator of order one in $\dot{\varphi}_{t}$ whose coefficients depend non-linearly on the metric $\omega_{t}$.

Proof. By the invariance of the Futaki character, if $\pi_{\omega} s_{\omega}$ is constant then so will be $\pi_{\tilde{\omega}} s_{\tilde{\omega}}$ for any other metric $\tilde{\omega}$ in $\mathfrak{M}_{\Omega, G}$ (see Section 4 of [20]). In that case, $X_{\Omega}$ is trivial and both sides of the expression in the statement are zero. The result follows.

So let us assume that $\pi_{t} s_{t}$ is not constant. Here and below we use the subscript $t$ to denote geometric quantities associated with $\omega_{t}$. Thus, the imaginary part of $X_{\Omega}=\partial_{\omega_{t}}^{\#} \pi_{t} s_{t}$ is a non-trivial Killing vector field, and in the construction of the projection map above, we can choose a basis $\left\{X_{j}\right\}$ for $\mathfrak{z}_{0}$ such that $X_{\Omega}=\partial_{\omega_{t}}^{\#}\left(\pi_{t} s_{t}\right)=J X_{1}+i X_{1}=X_{\Omega}$. Hence,

$$
\left.\pi_{t} s_{t}=2 i G_{t} \bar{\partial}_{t}^{*}\left(\omega_{t}\right\lrcorner X_{\Omega}\right)+s_{0}
$$

where $s_{0}$ is the projection (2.3) of $s$ onto the constants, a function that depends on $\Omega$ and $J$ but not on the particular choice of metric in $\mathfrak{M}_{\Omega, G}$.

By the Kähler identity $\bar{\partial}_{t}^{*}=-i\left[\Lambda_{t}, \partial\right]$, we conclude that

$$
\pi_{t} s_{t}=2 G_{t} \Lambda_{t} \partial\left(\omega_{t} \downarrow X_{\Omega}\right)+s_{0}
$$

and, therefore,

$$
\frac{d}{d t} \pi_{t} s_{t}=2 G_{t} \Lambda_{t} \partial\left(\dot{\omega}_{t} \downarrow X_{\Omega}\right)+2 G_{t} \dot{\Lambda}_{t} \partial\left(\omega_{t} \downarrow X_{\Omega}\right)+2 \dot{G}_{t} \Lambda_{t} \partial\left(\omega_{t} \downarrow X_{\Omega}\right) .
$$

The last two terms in the expression above cancel each other out. Indeed, $\omega_{t} \downarrow X_{\Omega}=$ $-i \bar{\partial}\left(\pi_{t} s_{t}\right)$ and computing the derivative of $\dot{\Lambda}_{t}$ in terms of $\dot{\varphi}_{t}$, we see that $2 G_{t} \dot{\Lambda}_{t} \partial\left(\omega_{t}-X_{\Omega}\right)=2 G_{t}\left(i \partial \bar{\partial} \dot{\varphi}_{t}, i \partial \bar{\partial}\left(\pi_{t} s_{t}\right)\right)_{t}$. On the other hand, the differential of the Green's operator is given by $-G_{t} \dot{\Delta}_{t} G_{t}$, and we obtain that $\left.2 \dot{G}_{t} \Lambda_{t} \partial\left(\omega_{t}\right\lrcorner X_{\Omega}\right)=$ $2 G_{t} \dot{\Delta}_{t} G_{t} \Lambda_{t} i \partial \bar{\partial}\left(\pi_{t} s_{t}\right)=-G_{t} \dot{\Delta}_{t}\left(\pi_{t} s_{t}\right)=-2 G_{t}\left(i \partial \bar{\partial} \dot{\varphi}_{t}, i \partial \bar{\partial}\left(\pi_{t} s_{t}\right)\right)_{t}$.

Since the real and imaginary parts of $X_{\Omega}$ are Killing vector fields and the metric potential $\varphi_{t}$ is $G$-invariant, we have that $X_{\Omega}\left(\dot{\varphi}_{t}\right)=0$, and so $\left.\partial \dot{\varphi}_{t}\right\lrcorner X_{\Omega}=$ $\left(\partial \dot{\varphi}_{t}, i \partial\left(\pi_{t} s_{t}\right)\right)_{t}$ is orthogonal to the constants. On the other hand, since $X_{\Omega}$ is holomorphic, we have that $\dot{\omega}_{t} \downarrow X_{\Omega}=i \partial \bar{\partial} \dot{\varphi}_{t} \downarrow X_{\Omega}=-i \bar{\partial}\left(\partial \dot{\varphi}_{t} \downarrow X_{\Omega}\right)$. Hence, $2 G_{t} \Lambda_{t} \partial\left(\dot{\omega}_{t} \downarrow X_{\Omega}\right)=-2 G_{t} \Lambda_{t} i \partial \bar{\partial}\left(\partial \dot{\varphi}_{t} \downarrow X_{\Omega}\right)$, and the desired result follows now because $G_{t}$ is the inverse of the Laplacian in the complement of the constants. 
Given any Kähler metric $g$ in $\mathfrak{M}_{\Omega, G}$, we have seen that the extremal vector field $X_{\Omega}$ of the class can be written as $X_{\Omega}=\partial_{g}^{\#}\left(\pi_{g} s_{g}\right)$. Thus, the critical points of $\pi_{g} s_{g}$ corresponds to zeroes of $X_{\Omega}$, and are therefore, independent of $g$. We may use the Lemma above to strengthen this assertion a bit, and derive the following remarkable consequence. This result is reminiscent of the convexity theorem on the image of moment mappings [1,9].

Theorem 3.3. Let $\omega$ be any metric in $\mathfrak{M}_{\Omega, G}$ and consider the function $\pi_{\omega} s_{\omega}$ obtained by projection of the scalar curvature onto the space of real holomorphy potentials. Then the range of $\pi_{\omega} s_{\omega}$ is a closed interval on the real line that only depends on the class $\Omega$ and the complex structure $J$, but not on the particular choice of metric $\omega \in \mathfrak{M}_{\Omega, G}$.

Proof. Let $\omega_{t}=\omega+i \partial \bar{\partial} \varphi_{t}$ be a path in $\mathfrak{M}_{\Omega, G}$. By Lemma 3.2, we have that

$$
\frac{d}{d t} \pi_{t} s_{t}=\left(\partial \dot{\varphi}_{t}, \partial\left(\pi_{t} s_{t}\right)\right)_{t} .
$$

Since the maximum and minimum of $\pi_{t} s_{t}$ occur at critical points, this expression shows that these extrema values do not change with $t$. The result follows because $\mathfrak{M}_{\Omega, G}$ is path connected.

The projection $\pi_{g}$ onto holomorphy potentials has an appropriate lift $\Pi_{g}$ to the level of $G$-invariant $(1,1)$ forms, which we discuss now. We denote by $\wedge_{k, G}^{1,1}$ the space of real forms of type $(1,1)$ that are invariant under $G$ and of class $L_{k}^{2}$.

Lemma 3.4. Given any G-invariant metric $\tilde{\omega}$, there exists a uniquely defined continuous projection map

$$
\Pi_{\tilde{\omega}}: \wedge_{k+2, G}^{1,1} \mapsto \wedge_{k+2, G}^{1,1},
$$

that intertwines the trace with the projection map $\pi_{\tilde{\omega}}$ in (3.2), and it is such that $\eta-\Pi_{\tilde{\omega}} \eta$ is cohomologous to zero for all $\eta \in \wedge_{k+2, G}^{1,1}$. For metrics $\tilde{\omega}$ as in (3.1), the map $\varphi \mapsto \Pi_{\tilde{\omega}}$ from $L_{k+4, G}^{2}$ to $\operatorname{End}\left(\wedge_{k+2, G}^{1,1}\right)$ is smooth.

Proof. Let $\eta \in \wedge_{k+2, G}^{1,1}$. Since $\Pi_{\tilde{\omega}} \eta$ must be of the form $\eta+i \partial \bar{\partial} f$ for some realvalued function $f$, the intertwining property of the projection and trace gives that

$$
\operatorname{trace}_{\tilde{\omega}} \eta-\frac{1}{2} \Delta_{\tilde{\omega}} f=\pi_{\tilde{\omega}} \operatorname{trace}_{\tilde{\omega}} \eta,
$$

and so

$$
\Delta_{\tilde{\omega}} f=-2\left(\pi_{\tilde{\omega}}-1\right) \operatorname{trace}_{\tilde{\omega}} \eta .
$$

The right side of this expression is a $G$-invariant real-valued function in the complement of the constants. Thus, we can solve the equation for $f$ to obtain a real-valued function that is invariant under $G$. By the continuity properties of the map $\pi_{\tilde{\omega}}$ for metrics as in (3.1), we conclude that $\varphi \mapsto \Pi_{\tilde{\omega}}$ is a smooth map from a suitable neighborhood of the origin in $L_{k+4, G}^{2}$ to the real Hilbert space $\operatorname{End}\left(\wedge_{k+2, G}^{1,1}\right)$. 


\subsection{Extremal flow equation}

The projection operators $\pi_{g}$ and $\Pi_{g}$ are essential elements in our study of extremal Kähler metrics. To begin with, they lead to alternative characterizations of the extremality condition of a metric that are quite suitable for analytical purposes. Indeed, if $g$ is an extremal metric, it must be invariant under a maximal compact subgroup $G$ of $\operatorname{Aut}(M, J)$ and we have that

$$
\rho_{g}=\Pi_{g} \rho_{g} .
$$

Conversely, any metric $g$ that is $G$-invariant and satisfies this equation must be extremal. Evidently, this tensorial equation for the $G$-invariant metric $g$ can be recast in terms of the equivalent scalar equation

$$
s_{g}=\pi_{g} s_{g},
$$

which also serves as a characterization of the extremal condition of $g$.

Notwithstanding the richer set-up, the definition of extremality embodied by (3.4) is analogous to the definition of an Einstein metric, and its algebraic nature is quite suitable and direct for analytical purposes. For instance, rephrasing the proof for Einstein metrics, we may use it to show that any $G$-invariant metric that is a $C^{1, \alpha}$ weak solution of the extremal equation in harmonic coordinates, must be in fact a smooth extremal Kähler metric (compare this statement with Lemma 1 in [13]).

However, the key use we shall make of these projections will be to define the extremal Kähler flow, a tool intended to govern the improvement of an initial representative of the class $\Omega$ towards one that is extremal.

The idea of using good flows to better geometric quantities was originally used by Eells and Sampson [6] in another context, and reconsidered by Hamilton [11] in his definition of the Ricci flow. In our case, we are given a metric in $\mathfrak{M}_{\Omega, G}$ and try to improve it by means of a non-linear pseudo-differential heat equation, requiring the velocity of the curve to equal the component of the Ricci curvature that is perpendicular to the image of $\Pi$.

More precisely, we fix a maximal compact subgroup $G$ of the automorphism group of $(M, J)$, and work on $\mathfrak{M}_{\Omega, G}$, the space of all $G$-invariant Kähler forms that represent $\Omega$. Given $\omega \in \mathfrak{M}_{\Omega, G}$, we consider a path $\omega_{t}$ of Kähler metrics that starts at $\omega$ when $t=0$ and obeys the flow equation $\partial_{t} \omega_{t}=-\rho_{t}+\Pi_{t} \rho_{t}$. Since $-\rho_{t}+\Pi_{t} \rho_{t}$ is cohomologous to zero and $G$-invariant, for as long as the solution exists, we will have that $\omega_{t} \in \mathfrak{M}_{\Omega, G}$. Thus, our evolution equation is given by the initial value problem

$$
\begin{aligned}
\partial_{t} \omega_{t} & =-\rho_{t}+\Pi_{t} \rho_{t}, \\
\omega_{0} & =\omega .
\end{aligned}
$$

Critical points of this equation correspond precisely to extremal metrics, those that satisfy (3.4).

In the same manner as (3.4) has the alternative scalar description (3.5), we may reformulate (3.6) as an scalar equation. If $\omega_{t}=\omega+i \partial \bar{\partial} \varphi_{t}$, we have that 
$\Pi_{t} \rho_{t}-\rho_{t}=i \partial \bar{\partial} G_{t}\left(s_{t}-\pi_{t} s_{t}\right)$, where $G_{t}$ is the Green's operator of the metric $\omega_{t}$. By compactness of $M$, we see that the deformation potential $\varphi_{t}$ evolves according to

$$
\begin{aligned}
\partial_{t} \varphi_{t} & =G_{t}\left(s_{t}-\pi_{t} s_{t}\right), \\
\varphi_{0} & =0 .
\end{aligned}
$$

A critical point of (3.7) is given by a metric for which $G_{\omega}\left(s_{\omega}-\pi_{\omega} s_{\omega}\right)=0$. Since $s_{\omega}-\pi_{\omega} s_{\omega}$ is orthogonal to the constant, this condition is precisely the extremality condition (3.5).

\subsection{General properties of the extremal flow}

We begin by making a rather expected observation.

Proposition 3.5. Let $\omega_{t}$ be a solution of the initial value problem (3.6). If $d \mu_{t}$ is the volume form, we have that

$$
\frac{d}{d t} d \mu_{t}=\frac{1}{2}\left(\pi_{t} s_{t}-s_{t}\right) d \mu_{t}
$$

In particular, the volume of $\omega_{t}$ is constant.

Proof. The volume form is given by

$$
d \mu_{t}=\frac{\omega_{t}^{n}}{n !}
$$

Differentiating with respect to $t$, we obtain:

$$
\frac{d}{d t} d \mu_{t}=\frac{1}{(n-1) !} \omega_{t}^{n-1} \wedge \dot{\omega}_{t}=\frac{1}{(n-1) !} \omega_{t}^{n-1} \wedge\left(\Pi_{t} \rho_{t}-\rho_{t}\right)=\frac{1}{2}\left(\pi_{t} s_{t}-s_{t}\right) d \mu_{t},
$$

as desired. Notice that this form of maximal rank is exact.

Our next results address the plausible existence of fixed points or periodic solutions of the flow equation.

Observe that (3.6) is invariant under the group of diffeomorphism that preserve the complex structure $J$. An extremal soliton is a solution that changes only by such a diffeomorphism. Then, there must be a holomorphic vector field $V=\left(V^{i}\right)$ such that $V_{i, \bar{j}}+V_{\bar{j}, i}=\Pi \rho_{i \bar{j}}-\rho_{i \bar{j}}$. If the vector field $V$ has a holomorphy potential $f$, we refer to the pair $(g, V)$ as a gradient extremal soliton.

Proposition 3.6. There are no extremal solitons other than extremal metrics.

Proof. Suppose we have an extremal gradient soliton $(g, V)$ defined by a holomorphic potential $f$. Then

$$
i \partial \bar{\partial} f=\Pi \rho-\rho,
$$

and therefore,

$$
f=G_{g}(s-\pi s) .
$$


This implies that $\Delta f=s-\pi s$ and since $\Delta$ is a real operator, the holomorphy potential $f$ must be real. But $f$ is a holomorphy potential, so it is $L^{2}$-orthogonal to $s-\pi s$. Hence,

$$
\|\nabla f\|^{2}=\int f \Delta f d \mu_{g}=\int f(1-\pi) s d \mu=0 .
$$

Thus, $f$ is constant, and therefore, necessarily zero.

Thus, a non-trivial soliton, if any, must be given by a holomorphic vector field $V$ that is not a gradient. The set of all such vector fields forms an Abelian subalgebra of the algebra of holomorphic vector fields. The group of diffeomorphism they generate must be in the maximal compact subgroup $G$ of isometries of the metric. This vector field does not change the metric and so $\omega_{t}=(\exp (t V))^{*} \omega=\omega$. Hence, $\dot{\omega}_{t}=0=\Pi \rho-\rho$, and the metric is extremal.

Remark 3.7. Evidently, the compactness of $M$ plays an important rôle in this argument that rules out extremal solitons other than extremal metrics. They do exist in the non-compact case, where they give rise to certain points in the moduli space of these metrics.

We now show that the evolution equation (3.6) is essentially the gradient flow of the $K$-energy, function that also serve to characterize extremal Kähler metrics [17]. Indeed, given two elements $\omega_{0}$ and $\omega_{1}$ of $\mathfrak{M}_{\Omega, G}$, there exists a $G$-invariant function $\varphi$, unique modulo constants, such that $\omega_{1}=\omega_{0}+i \partial \bar{\partial} \varphi$. Let $\varphi_{t}$ be a curve of $G$-invariant functions such that $\omega_{t}=\omega_{0}+i \partial \bar{\partial} \varphi_{t} \in \mathfrak{M}_{\Omega, G}$ and $\omega(0)=\omega_{0}$, $\omega(1)=\omega_{1}$. We set

$$
M\left(\omega_{0}, \omega_{1}\right)=-\int_{0}^{1} d t \int_{M} \dot{\varphi}_{t}\left(s_{t}-\pi_{t} s_{t}\right) d \mu_{t},
$$

where $s_{t}$ and $d \mu_{t}$ are the scalar curvature and volume form of the metric $\omega_{t}, \pi_{t}$ is the projection (3.2) onto the space of $G$-invariant holomorphic potentials associated with this same metric, and $\dot{\varphi}_{t}=\frac{d \varphi_{t}}{d t}$. This definition is independent of the curve $t \rightarrow \varphi_{t}$ chosen.

Fix $\omega_{0} \in \mathfrak{M}_{\Omega, G}$. The $K$-energy is defined to be

$$
\begin{aligned}
\mathfrak{M}_{\Omega, G} & \stackrel{\kappa}{\longrightarrow} \mathbb{R} \\
\omega & \rightarrow M\left(\omega_{0}, \omega\right) .
\end{aligned}
$$

We have (see Proposition 2 in [17]) that

$$
\frac{d}{d t} \kappa\left(\omega_{t}\right)=-\int_{M} \dot{\varphi}_{t}\left(s_{t}-\pi_{t} s_{t}\right) d \mu_{t} .
$$

Thus, up to the action of the non-negative Green's operator, the gradient of $\kappa$ is given by the right-side of (3.7). Indeed, along flow paths, the $t$-derivative of $\kappa\left(\omega_{t}\right)$ is just the negative $L_{\omega_{t}}^{2}$-inner product of $s_{t}-\pi_{t} s_{t}$ and $G_{t}\left(s_{t}-\pi_{t} s_{t}\right)$, respectively. 
Proposition 3.8. Let $\omega_{t}$ be a solution of the initial value problem (3.6). Then

$$
\frac{d}{d t} \kappa\left(\omega_{t}\right)=-\int_{M}\left(s_{t}-\pi_{t} s_{t}\right) G_{t}\left(s_{t}-\pi_{t} s_{t}\right) d \mu_{t} .
$$

We use this result and the non-negativity of the Green's operator to rule out nontrivial periodic orbits of the flow (3.6). This must be done because in addition to the ones studied above, the flow equation is also invariant under the one-parameter group of homotheties, where time scales like the square of the distance. In principle, such an invariance could give rise to periodic orbits.

Proposition 3.9. The only periodic orbits of the flow equation (3.6) are its fixed points, that is to say, the extremal metrics (if any) in $\mathfrak{M}_{\Omega, G}$.

Proof. Consider the $K$-energy suitably normalized by a volume factor to make it scale invariant. If there is a loop solution $\omega_{t}$ of (3.6) for $t \in\left[t_{1}, t_{2}\right]$, since the volume remains constant, we will have that $\kappa\left(\omega_{t_{1}}\right)=\kappa\left(\omega_{t_{2}}\right)$. By the previous proposition, since $G_{t}$ is a non-negative operator, we conclude that $G_{t}\left(s_{t}-\pi_{t} s_{t}\right)=0$ on this time interval. This says that $\omega_{t}$ is extremal for each $t$ on the interval, and so the right side of the evolution equation is zero. Thus, the loop is trivial, a fixed point of the flow.

We end this section by showing that the functional (2.4) decreases along the flow (3.6). This should be clear from the way the equation was set-up, or at the very least, expected.

Proposition 3.10. Let $\omega_{t}$ be a path in $\mathfrak{M}_{\Omega, G}$ that solves the flow equation (3.6). Then

$$
\frac{d}{d t} \Phi_{\Omega}\left(\omega_{t}\right)=-4 \int\left(s_{t}-\pi_{t} s_{t}\right) L_{t} G_{t}\left(s_{t}-\pi_{t} s_{t}\right) d \mu_{t} \leq 0,
$$

and the equality is achieved if and only if $\omega_{t}$ is extremal. In this expression, $L_{t}=$ $\left(\bar{\partial} \partial^{\#}\right)^{*}\left(\bar{\partial} \partial^{\#}\right)$ and $G_{t}$ is the Green's operator if $\omega_{t}$.

Proof. Given any variation of the metric with potential function $\varphi$, we know that

$$
\frac{d}{d t} \Phi\left(\omega_{t}\right)=-4 \int s L_{t} \dot{\varphi} d \mu_{t}
$$

But $\dot{\varphi}=G_{t}\left(s_{t}-\pi_{t} s_{t}\right)$, and since $\pi_{t} s_{t}$ is a holomorphy potential and $L_{t}$ is selfadjoint, we see that

$$
\frac{d}{d t} \Phi\left(\omega_{t}\right)=-4 \int\left(s_{t}-\pi_{t} s_{t}\right) L_{t} G_{t}\left(s_{t}-\pi_{t} s_{t}\right) d \mu_{t} .
$$

Both $L_{t}$ and $G_{t}$ are non-negative elliptic operators. Thus, $L_{t} G_{t}$ is elliptic and, furthermore, its spectrum is contained in $[0, \infty)$. For if $L_{t} G_{t} \varphi=\lambda \varphi$, we have that $G_{t} L_{t} G_{t} \varphi=\lambda G_{t} \varphi$, and taking the $L^{2}$-inner product with $\varphi$ itself, we conclude that $\lambda$ must be real and non-negative. Therefore, the the expression above for the 
derivative of $\Phi_{\Omega}$ along flow paths must be non-positive. If it reaches the value zero at some $t$, then we must have that $f_{t}=G_{t}\left(s_{t}-\pi_{t} s_{t}\right)$ is a holomorphy potential and $\Delta_{t} f=\left(1-\pi_{t}\right) s_{t}$ is an element of the image of $1-\pi_{t}$. Thus, $f_{t}$ is $L^{2}$-orthogonal to $\left(1-\pi_{t}\right) s_{t}$. An integration by parts argument yields then that $\nabla_{t} f_{t}$ must be zero, and so the function $f_{t}$ is a constant, which is necessarily zero. Thus, $s_{t}=\pi_{t} s_{t}$ and the metric $\omega_{t}$ is extremal.

It is clear that we could have used the function $\Phi_{\Omega}$ in the rôle that $\kappa$ played when proving that the flow does not have periodic orbits other than its fixed points. In fact, it is better to work with $\Phi_{\Omega}$ itself. For we do not know if $\kappa$ is in general bounded below on $\mathfrak{M}_{\Omega, G}$, but the energy functional $\Phi_{\Omega}$ has that property indeed. If the solution to the flow equation were to exist for all $t \in[0, \infty)$, the monotonicity result above would lead us to expect that, as $t \rightarrow \infty$, the sequence $\omega_{t}$ should be getting closer and closer to an extremal metric. We shall make several remarks about this possibility later on, but our discussion here of both, $\kappa$ and $\Phi_{\Omega}$, serves to show the similarities in their behaviour along solution paths to the the extremal flow equation. This is rather natural since they are both functions that can be used to characterize extremal Kähler metrics.

\section{The linearized flow equation}

Consider a family of metrics in $\mathfrak{M}_{\Omega, G}$ of the form $\omega_{t}(v)=\omega_{\varphi}+i \partial \bar{\partial} \alpha(t, v)$, with $\alpha(t, 0)=0$. We set $\beta=\beta_{t}=\left.\frac{d \alpha(t, v)}{d v}\right|_{v=0}$. The linearization of (3.7) at $\omega_{\varphi}$ in the direction of $\beta$ is given by

$$
\partial_{t} \beta_{t}=\left.\frac{d}{d v}\left(G_{(t, v)}\left(\left(1-\pi_{(t, v)}\right) s_{(t, v)}\right)\right)\right|_{v=0} .
$$

Of course, the argument of the $v$-differentiation in the right side involves quantities associated with the metric $\omega_{t}(v)$.

In the remaining part of this section we use the subscript $\varphi$, or no subscript at all, to denote geometric quantities associated with the metric $\omega_{\varphi}$.

We have that

$$
\left.\frac{d s_{(t, v)}}{d v}\right|_{v=0}=-\frac{1}{2} \Delta_{\varphi}^{2} \beta-2\left(\rho_{\varphi}, i \partial \bar{\partial} \beta\right)_{\varphi}
$$

Since the variation of the Green's operator is $-G_{\varphi}\left(\frac{d}{d v} \Delta_{(t, v)}\right) G_{\varphi}$ (keep in mind that this operator needs to be applied only to $s-\pi s$, a function that is orthogonal to the constants), using the relation between $\rho_{\varphi}$ and $\Pi_{\varphi} \rho_{\varphi}$, we obtain that

$$
\partial_{t} \beta=-\frac{1}{2} \Delta_{\varphi} \beta-2 G_{\varphi}\left(\Pi_{\varphi} \rho_{\varphi}, i \partial \bar{\partial} \beta\right)_{\varphi}-G_{\varphi}\left(\left.\frac{d}{d v} \pi_{(t, v)} S_{(t, v)}\right|_{v=0}\right) .
$$

By Lemma 3.2, we may write this as

$$
\partial_{t} \beta=-\frac{1}{2} \Delta_{\varphi} \beta-2 G_{\varphi}\left(\Pi_{\varphi} \rho_{\varphi}, i \partial \bar{\partial} \beta\right)_{\varphi}-G_{\varphi}\left(\partial_{\varphi}^{\#} \beta, X_{\Omega}\right)_{\varphi},
$$


where $X_{\Omega}=\partial_{\varphi}^{\#}\left(\pi_{\varphi} s_{\varphi}\right)$ is the holomorphic vector field of the class $\Omega$. Notice that

$$
P_{\varphi}(\beta):=G_{\varphi}\left(\partial_{\varphi}^{\#} \beta, X_{\Omega}\right)_{\varphi}
$$

is a pseudo-differential operators of order -1 in $\beta$ whose coefficients depend nonlinearly on the coefficients of the metric $\omega_{\varphi}$.

We summarize our discussion with the following:

Theorem 4.1. Let $(M, J, \Omega)$ be a polarized Kähler manifold and let $G$ be a maximal compact subgroup of Aut $(M, J)$. The extremal flow equation (3.7) (or equivalently, (3.6)) in $\mathfrak{M}_{\Omega, G}$ is a non-linear pseudo-differential parabolic equation.

Remark 4.2. For a generic manifold $(M, J)$ the non-trivial holomorphic fields, if any, have no zeroes, and the space of real holomorphy potentials reduces to the constant functions. Under that hypothesis, the pseudo-differential term of order -1 in the right side of the linearized flow equation (4.1) vanishes, and the equation reduces to

$$
\partial_{t} \beta_{t}=-\frac{1}{2} \Delta_{\varphi} \beta-2 G_{\varphi}\left(\Pi_{\varphi} \rho_{\varphi}, i \partial \bar{\partial} \beta\right)_{\varphi} .
$$

This is still a pseudo-differential equation, a zero-order perturbation of pseudodifferential type of the standard time dependent heat equation. Thus, even for generic complex manifolds of Kähler type, the pseudo-differential nature of our flow equation remains in place.

Remark 4.3. Even when the cohomology class $\Omega$ under study is the canonical class $c_{1}$ (which a fortiori must then have a sign), the extremal and Ricci flow do not necessarily coincide with one another. That will only be the case if we know a priori that $\pi s$ is a constant, which as we saw earlier, is a rather non-trivial condition to impose and only happens if the Futaki character of the canonical class vanishes. Such a restrictive condition fails, for instance, when the manifold is the blow up of $\mathbb{C P}^{2}$ at one or two points.

We now introduced an approximate linearized equation whose solution is needed in our study of local solvability of (3.7). In order to do so, we make some preliminary observations.

Let $T$ be a positive real number to be determined later and set $I=[0, T]$. A scale $\mathcal{Y}=\left\{\mathcal{Y}_{j}\right\}_{j \geq 0}$ of Banach spaces is a countable family of complete normed spaces such that $\mathcal{Y}_{j} \supset \mathcal{Y}_{j+1}$ and each $\mathcal{Y}_{j}$ is dense in $\mathcal{Y}_{0}$. Given one such, we define

$$
C_{(j, k)}(I ; \mathcal{Y})=C^{0}\left(I ; \mathcal{Y}_{j}\right) \cap \cdots \cap C^{j-k}\left(I ; \mathcal{Y}_{k}\right),
$$

and provide it with the norm

$$
\|v\|_{j, k}=\sup _{t \in I}\left\{\sup _{0 \leq r \leq j-k}\left\{\left\|\partial_{t}^{r} v(t)\right\|_{j-r}\right\}\right\} .
$$

In what follows, where we shall consider metrics of the form $\omega_{t}=\omega+i \partial \bar{\partial} \varphi_{t}$ for path of functions $\varphi_{t}$ that begin at 0 when $t=0$, we shall always use the scale of Sobolev spaces

$$
\mathcal{Y}_{j}=L_{2 j, G}^{2}(M)
$$


as defined by the background metric $\omega$. When $t$ varies on the interval $[0, T]$, if we choose $T$ sufficiently small, all the metrics $\omega_{t}$ will be equivalent, and the Sobolev spaces defined by them will be equivalent to each other, with equivalent norms. We let the Sobolev order jump by 2 because the operator $F(\varphi)$ in the right side of (3.7),

$$
F(\varphi):=G_{t}\left(s_{t}-\pi_{t} s_{t}\right)
$$

is of second order, the one reason for the peculiar definition of the scale $\mathcal{Y}_{j}$ we shall use. And recall that by the Sobolev embedding theorem, we know that $L_{k}^{2}(M)$ is a Banach algebra whenever $k>n$. Thus, for as long as the metric $\omega_{t}$ is equivalent to $\omega$, provided that $k>n$, we have a continuous mapping

$$
F: L_{k+4}^{2}(M) \mapsto L_{k+2}^{2}(M)
$$

Proposition 4.4. Assume that a solution $\varphi(t)$ of $(3.7)$ is in $C_{(k+1,0)}(I ; \mathcal{Y})$ on the interval I for some integer $k$ such that $2 k>n+2$. Then all the values of $\partial_{t}^{r} \varphi(t)$ $(1 \leq r \leq k+1)$ restricted to $t=0$ are completely determined and $\left.\partial_{t}^{r} \varphi(t)\right|_{t=0}:=$ $\varphi_{r} \in \mathcal{Y}_{k+1-r}=L_{2 k+2-2 r, G}^{2}(M)$.

Proof. The initial condition $\left.\varphi\right|_{t=0}$ is zero, and the equation itself sets the value of $\left.\partial_{t} \varphi\right|_{t=0}=F(0)=G_{\omega}\left(s_{\omega}-\pi_{\omega} s_{\omega}\right)$ that is evidently in $L_{2 k, G}^{2}(M)$.

The relation (4.1) for $\beta=\partial_{t} \varphi_{t}$ says that

$$
\frac{d}{d t} \beta=-\frac{1}{2} \Delta_{\varphi} \beta+P_{0}(\varphi) \beta
$$

where $P_{0}$ is a pseudo-differential operator of order zero whose coefficients depend on the coefficients of the metric $\omega_{\varphi}$ and its curvature tensor. Since $\varphi_{t} \in$ $C\left(I ; L^{2} 2 k(M)\right)$ and $2 k>n+2$, by the Sobolev embedding theorem, these coefficients are continuous functions. By regularity of pseudo-differential operators on Sobolev spaces, we obtain that $\partial_{t} \beta=\partial_{t}^{2} \varphi_{t} \in L_{2(k-1)}^{2}(M)$, which is still a continuous function because $2(k-1)>n$.

If we differentiate the expression above for $\beta=\partial_{t} \varphi_{t}$ with respect to $t$, we obtain

$$
\frac{d^{2}}{d t^{2}} \beta=-\frac{1}{2} \Delta_{\varphi} \frac{d}{d t} \beta-\frac{1}{2} L_{\varphi}(\beta) \beta+P_{0}(\varphi) \frac{d}{d t} \beta+P_{0, \varphi}(\beta) \beta,
$$

where $L_{\varphi}(\beta)$ and $P_{0, \varphi}(\beta)$ are the linearizations of $\Delta_{\varphi}$ and $P_{0}(\varphi)$ at $\varphi$ in the direction of $\partial_{t} \varphi$, respectively. The first is an operator of order two whose coefficients are continuous. By the metric dependence of $P_{0}(\varphi)$, the latter is a pseudo-differential operator of order zero whose coefficients are also continuous functions. Hence, $\partial_{t}^{2} \beta=\partial_{t}^{3} \varphi_{t} \in L_{2(k-2)}^{2}(M)$.

Iteration of the argument above yields that

$$
\partial_{t}^{r} \varphi=F_{r}\left(\varphi, \partial_{t} \varphi, \ldots, \partial_{t}^{r-1} \varphi\right)
$$


where $F_{r}$ is some operator whose coefficients depend upon the coefficients of the metric $\omega_{\varphi}=\omega+i \partial \bar{\partial} \varphi$. The desired result for the regularity of $\partial_{t}^{r} \varphi$ follows again using the Sobolev embedding theorem and the known regularity of the lower order time derivatives $\partial_{t}^{j} \varphi, 0 \leq j \leq r-1$.

Assume given Cauchy data $\varphi_{0}=0$ for (3.7) and let $\varphi_{r}=\left.\partial_{t}^{r} \varphi(t)\right|_{t=0}$ be the sequence of coefficients of the Taylor series of $\varphi(t)$ given by the proposition above. The Cauchy data $\varphi_{0}$ determines the sequence $\varphi_{r}, 1 \leq r \leq k+1$. We consider the metric space:

$$
W(I)=W^{k}(I)=\left\{\psi(t) \in C_{(k+1,0)}(I ; \mathcal{Y}):\left.\partial_{t}^{r} \psi(t)\right|_{t=0}=\varphi_{r}, 0 \leq r \leq k+1\right\} .
$$

This space is not empty, as can be seen by solving the Cauchy problem for a suitable parabolic

equation.

By a continuity argument, for any $\psi(t) \in W(I)$ the form $\omega_{\psi}=\omega+i \partial \bar{\partial} \psi(t)$ is positive provided that $t$ is sufficiently small. Hence, $\omega_{\psi}$ defines a Kähler metric. This metric is not smooth in general. However, if $2 k>n+2$, by the Sobolev embedding theorem, $\omega_{\psi}$ is at least $C^{2}$, and the operator in the right-hand side of (4.1) will make sense when $\psi$ plays the rôle of $\varphi$. Thus, we set

$$
P_{0}(\psi) b=-2 G_{\psi}\left(\Pi_{\psi} \rho_{\psi}, i \partial \bar{\partial} b\right)_{\psi}-G_{\psi}\left(\partial_{\psi}^{\#} b, X_{\Omega}\right)_{\psi} \cdot
$$

Then $P_{0}(\psi)$ is a pseudo-differential operator of order zero in $b$, whose coefficients depend upon the coefficients of the metric $\omega_{\psi}$ and its curvature tensor, all of which are continuous functions. For each $t$ on a time interval where all the metrics $\omega_{\psi}$ are uniformly equivalent, we have that

$$
-\frac{1}{2} \Delta_{\psi}+P_{0}(\psi): L_{2, G}^{2}(M) \rightarrow L_{0, G}^{0}(M)=L^{2}(M)
$$

continuously. We consider the equation

$$
\frac{d}{d t} b=-\frac{1}{2} \Delta_{\psi} b+P_{0}(\psi) b,
$$

whose Cauchy problem will be studied in the next section. We shall refer to it as the approximate linearized equation, the reasons being —we hope-clear at this point.

We end this section with the following:

Proposition 4.5. Let $\varphi_{1}$ be the Cauchy data for (4.5). If $b(t) \in C_{(k, 0)}(I, \mathcal{Y})$ is a solution, then $\left.\partial_{t}^{r} b\right|_{t=0}=\varphi_{r+1}, 0 \leq r \leq k$.

Proof. We have seen above that if $\varphi(t)$ satisfies (3.7), then

$$
\partial_{t}^{r} \varphi=F_{r}\left(\varphi, \partial_{t} \varphi, \ldots, \partial_{t}^{r-1} \varphi\right), \quad r \geq 2,
$$


where $F_{r}$ is some operator whose coefficients depend upon the coefficients of the metric $\omega_{\varphi}=\omega+i \partial \bar{\partial} \varphi$, and whose restriction to $t=0$ depends only on the sequence $\varphi_{0}, \varphi_{1}, \ldots, \varphi_{r-1}$. The approximate linearized equation (4.5) is obtained from the linearization of (3.7) given in (4.1), when we replace the rôle played by $\varphi(t)$ by that of $\psi(t)$. But $\psi(t)$ and $\varphi(t)$ have the same coefficients in their Taylor expansions up to order $k+1$. Therefore, the solution $b(t)$ to the Cauchy problem of (4.5) with data $b(0)=\varphi_{1}$ will have necessarily a Taylor series of order $k$ that agrees with the Taylor series of the solution to the Cauchy problem of linearized equation (4.1). The conclusion follows by Proposition 4.4.

\section{Local solvability of the extremal flow equation}

In this section we prove local time existence of solutions to the extremal flow (3.7). We do so by adapting to our situation a method of T. Kato for the solvability of abstract differential equations and non-linear problems [12]. The pseudo-differential nature of our linearized equation (4.1) makes the task harder. But, fortunately enough, the strictly pseudo-differential part of the equation is of lower order, and most of the analysis is based on that of the standard time-dependent heat equation.

\subsection{The Cauchy problem for the approximate linearized equation}

From now on, we take $k$ to be an integer such that $2 k>n+2$ and $\mathcal{Y}_{j}=L_{2 j, G}^{2}(M)$ as in the previous section. Given Cauchy data $\varphi_{0}=0$ for (3.7), Proposition 4.4 determines the sequence $\left\{\varphi_{j}\right\}_{j=0}^{k+1}$, and that in turn allows us to define the space $W(I)$ of (4.2). The interval $I=[0, T]$ will be determined later.

For $\psi \in W(I)$, we consider the metrics $\omega_{\psi}=\omega+i \partial \bar{\partial} \psi$ and the Cauchy problem of the approximate linearized equation (4.5). Notice that in that equation, $P_{0}(\psi)$ is given by (4.3), a pseudo-differential operator of order zero whose coefficients depend non-linearly on the coefficients of the metric $\omega_{\psi}$ and its curvature tensor.

Let $p(t, s)$ be the evolution operator of

$$
\frac{d}{d t} b=-\frac{1}{2} \Delta_{\psi} b .
$$

Thus, $p(t, s)$ is a two-parameter family of strongly continuous operators on $\mathcal{Y}_{0}$ and $\mathcal{Y}_{1}$, respectively, such that $p(t, s) p(s, r)=p(t, r), p(t, t)=1$, and for $b \in \mathcal{Y}_{1}$ we have

$$
\begin{aligned}
\partial_{t} p(t, s) b & =-\frac{1}{2} \Delta_{\psi(t)} p(t, s) b, \\
\partial_{s} p(t, s) b & =-\frac{1}{2} p(t, s) \Delta_{\psi(s)} b .
\end{aligned}
$$

This family of operators exists for $0 \leq s \leq t \leq T$, and their operator norm is bounded uniformly by a constant that only depends upon a bound on $I=[0, T]$ 
of the coefficients of $\omega_{\psi(t)}$. The function solving (4.5) with Cauchy data $\beta$ must satisfy the integral equation

$$
b(t)=p(t, 0) \beta+\int_{0}^{t} p(t, s) P_{0}(\psi(s)) b(s) d s .
$$

Consider the set of functions $b(t)$ in $C_{(1,0)}(I ; \mathcal{Y})=C\left(I ; \mathcal{Y}_{0}\right) \cap C^{1}\left(I ; \mathcal{Y}_{1}\right)$ such that $b(0)=\beta$. The right-hand side of the above expression defines an operator in this space,

$$
P: b \mapsto p(t, 0) \beta+\int_{0}^{t} p(t, s) P_{0}(\psi(s)) b(s) d s,
$$

and by the explicit form of the coefficients of $P_{0}(\psi)$ mentioned above, combined with the continuity of pseudo-differential operators on Sobolev spaces, we have that

$$
\|P b-P \tilde{b}\| \leq C T\|b-\tilde{b}\|,
$$

where $C$ is a constant that depends upon the $L^{\infty}$-norm of the coefficients of $\omega_{\psi(t)}$ and its curvature tensor on the time interval $I$. A fixed-point argument now yields the following result:

Theorem 5.1. Consider the Cauchy problem for (4.5) with Cauchy data $\left.b(t)\right|_{t=0} \in$ $\mathcal{Y}_{1}$. Then there exists $T$ such that this problem has a unique solution in $C_{(1,0)}(I ; \mathcal{Y})=$ $C\left(I ; \mathcal{Y}_{0}\right) \cap C^{1}\left(I ; \mathcal{Y}_{1}\right)$. The value of $T$ only depends on supremum norms of the coefficients of $\omega_{\psi(t)}$ and its curvature tensor.

Of course, the regularity of the solution in the above theorem can be improved if we start with a better initial condition. For that, observe that the coefficients of the operator $\Delta_{\psi}$ are curves in $C_{(k, 0)}(I ; \mathcal{Y})$, and consequently,

$$
\Delta_{\psi(t)}: L_{2 j}^{2}(M) \mapsto L_{2 j-2}^{2}(M), 1 \leq j \leq k,
$$

continuously. While the metrics remain equivalent, we can choose a uniform constant for the operator norm of these maps, and (5.1) holds for $b \in L_{2 j}^{2}(M)$ with $j$ 's in this range. Then we have:

Corollary 5.2. If the initial data $\left.b(t)\right|_{t=0}=\varphi_{1} \in \mathcal{Y}_{k}$, the solution to the Cauchy problem for $(4.5)$ belongs to $C_{(k, 0)}(I ; \mathcal{Y})=C\left(I ; \mathcal{Y}_{k}\right) \cap \cdots \cap C^{k}\left(I, \mathcal{Y}_{0}\right)$.

Proof. The arguments in the proof of the theorem and the remarks made above show that we now have a solution $b(t)$ to the Cauchy problem for (4.5) that is in $C\left(I ; \mathcal{Y}_{k}\right) \cap C^{1}\left(I ; \mathcal{Y}_{k-1}\right)$. This solution satisfies (5.2) with $\beta=\varphi_{1}$.

We can differentiate repeatedly the identity (4.5) in order to show that the regularity of $b(t)$ with this initial condition can be improved. Notice that the coefficients of the second order operators $d_{t}^{r} \Delta_{\psi(t)}, 1 \leq r \leq k-1$, are curves in $C\left(I ; L_{2 k-2 r}^{2}\right)$, and so we have $d_{t}^{r} \Delta_{\psi(t)} \in C\left(I ; \mathcal{L}\left(\mathcal{Y}_{j+r+1}, \overline{\mathcal{Y}}_{j}\right)\right)$ for $0 \leq j \leq k-1-r$. Here, $\mathcal{L}(X, Y)$ is the space of linear bounded operators from $X$ to $Y$, and the assertion follows because in the stated range, $L_{2 k-2 r}^{2} \cdot L_{2 j+2 r}^{2} \subset L_{2 j}^{2}$. This suffices to conclude 
that the contributions to $d_{t}^{l+1} b$ arising from $d_{t}^{l} \Delta_{\psi} b$ are in $L_{2 k-2 l-2}^{2}$ if we already know that $b \in C_{(k, l)}(I ; \mathcal{Y})$.

The analysis of the contributions to $d_{t}^{l+1} b$ arising from $d_{t}^{l}\left(P_{0}(\psi) b\right)$ is similar. This time, the coefficients of the operators $d_{t}^{r}\left(P_{\psi(t)}\right)$ are curves in $C\left(I ; H^{2 k-2 r-2}\right)$, one degree worse than those of $d_{t}^{r} \Delta_{\psi(t)}$, but the operators are of pseudo-differentials of order zero instead. The desired improved regularity follows by the same arguments as the ones in the previous paragraph.

\subsection{An elliptic equation for $\gamma-F$}

Let us recall that $F(\varphi)=G_{\varphi}\left(s_{\varphi}-\pi_{\varphi} s_{\varphi}\right)$ is the second order non-linear operator defined by the right-hand side of (3.7). The derivative $L_{\psi}$ of this map at a general point $\psi$ in $\mathcal{Y}_{k+1}$ was computed in Section 4 and equals the operator in the right-hand side of (4.1):

$$
L_{\psi} b=-\frac{1}{2} \Delta_{\psi} b-2 G_{\psi}\left(\Pi_{\psi} \rho_{\psi}, i \partial \bar{\partial} b\right)_{\psi}-G_{\psi}\left(\partial_{\psi}^{\#} b, X_{\Omega}\right)_{\psi}
$$

Since the top part of this linearization is the negative operator $-\frac{1}{2} \Delta_{\psi}$, while the lower order term is a pseudo-differential operator of order zero, coercive estimates for this linearization imply that $\lambda-L_{\psi}$ is an invertible operator as a map, say, from $\mathcal{Y}_{1}$ to $\mathcal{Y}_{0}$, for a sufficiently large constant $\lambda$.

Let us then take a constant $\lambda$, and consider the non-linear elliptic map

$$
\begin{aligned}
\mathcal{Y}_{k+1} & \longrightarrow \mathcal{Y}_{k} \\
\varphi & \mapsto \lambda \varphi-F(\varphi) .
\end{aligned}
$$

We remind the reader here of the sequence $\left\{\varphi_{r}\right\}$ given by Proposition 4.4 , whose first element is $\varphi_{0}=0$.

Proposition 5.3. For $\lambda$ sufficiently large, there are neighborhoods $\mathcal{O}$ and $\mathcal{V}$ of $\varphi_{0}$ and $-\varphi_{1}$ in $\mathcal{Y}_{k+1}$ and $\mathcal{Y}_{k}$, respectively, such that the restriction of (5.4) to $\mathcal{O}$ is an isomorphism onto $\mathcal{V}$.

Proof. This is a consequence of the Inverse Function Theorem. Indeed, the linearization $\lambda-L_{0}$ is an invertible operator from $\mathcal{Y}_{1}$ to $\mathcal{Y}_{0}$. Hence, if $f \in \mathcal{Y}_{k}$, there exists an element $b \in \mathcal{Y}_{1}$ that satisfies the equation

$$
\left(\lambda-L_{0}\right) b=f
$$

We just need now to show that the regularity of $b$ can be improved.

For that observe that the above identity says that the image of $b$ under $L_{0}$ is in $\mathcal{Y}_{1}$, and by the regularity properties of $\lambda-L_{0}$, we must have $b \in \mathcal{Y}_{2}$. By iteration of this argument, we conclude that $b \in \mathcal{Y}_{k+1}$, and so, $b$ is an element of the tangent space of $\mathcal{Y}_{k+1}$ at 0 . The desired result follows. 
Corollary 5.4. Let $\psi \in \mathcal{Y}_{k}$ be sufficiently closed to $-\varphi_{1}$. Then, for large $\lambda$, the equation

$$
\lambda \varphi-F(\varphi)=\psi
$$

has a solution $\varphi \in \mathcal{Y}_{k+1}$. The solution is unique if it is required to be close enough to $\varphi_{0}=0$.

In the sequel, we let $D=D^{k}$ be the open neighborhood of $\varphi_{0}$ in $\mathcal{Y}_{k+1}$ where the operator $F(\varphi)$ is defined and smooth.

\subsection{A fixed point argument: local solvability of the non-linear equation}

Proceeding by analogy with [12], we define $E_{\varphi_{0}}(I)$ to be the set of curves $\psi(t) \in$ $W^{k}(I) \subset C_{(k+1,0)}(I ; \mathcal{Y})$ such that

$$
\left\|\partial_{t}^{l} \psi(t)-\varphi_{l}\right\|_{k+1-l} \leq R, l=0, \ldots, k, t \in I,
$$

for some positive constant $R$. The value of $R$ is chosen so that the ball in $\mathcal{Y}_{k+1}$ with center $\varphi_{0}$ and radius $R$ is contained in the domain $D$ where the operator $F(\varphi)$ is defined. This space is not empty for some $R>0$ and some $I=[0, T]$.

By the form (5.3) of the linearization of $F(\varphi)$ at $\psi$, we may conclude that if $\psi_{1}$ and $\psi_{2}$ are elements of $\mathcal{Y}_{k+1}$, then the operator norm, as a map from $\mathcal{Y}_{k}$ to $\mathcal{Y}_{0}$, satisfies the estimate

$$
\left\|L_{\psi_{1}}-L_{\psi_{2}}\right\|_{k, 0} \leq C\left\|\psi_{1}-\psi_{2}\right\|_{1},
$$

for some constant $C$. Indeed, the top part of $L_{\psi}$ in (5.3) is half of the Laplacian, and its lower order part is a zero-order pseudo-differential operator with nicely behaved coefficients. Then the regularity of pseudo-differential operators on Sobolev spaces yields the assertion made.

We now define a key mapping in our proof of the local time existence to the extremal flow. Let $\psi(t)$ be an element of $E_{\varphi_{0}}(I)$, and consider the solution $b(t)$ of (4.5) given in Theorem 5.1, with initial data $\varphi_{1}$. We then solve the equation

$$
\lambda \varphi-F(\varphi)=-b(t)+\lambda\left(\varphi_{0}+\int_{0}^{t} b(u) d u\right),
$$

where we use a real number $\lambda$ such that, if $L_{0}$ is the linearization (5.3) of $F(\varphi)$ at $\varphi=\varphi_{0}$, then $\lambda-L_{0}$ is an isomorphism.

We think of this as a stationary equation in $\varphi$, that is solved for each $t \in I$. Since for $t$ sufficiently small the right-hand side of the equation lies in a neighborhood of $-\varphi_{1}$, Corollary 5.4 applies to produce a solution $\varphi(t)$ in a neighborhood of $\varphi_{0}$.

The following two results are the versions of Proposition 7.4 and Proposition 7.6 in [12] adapted to our problem. We give proofs here for the sake of completeness. 
Proposition 5.5. For sufficiently small $t$, (5.5) has a unique solution $\varphi(t)$ in a neighborhood of $\varphi_{0}$ in $D \subset \mathcal{Y}_{k+1}$, with $\varphi(0)=\varphi_{0}=0$. Furthermore, $\varphi(t) \in$ $C_{(k+1,0)}(I ; \mathcal{Y})$ and $\left.\partial_{t}^{r} \varphi(t)\right|_{t=0}=\varphi_{r}, 0 \leq r \leq k$ provided $T$ is chosen sufficiently small, uniformly in $\psi \in E_{\varphi_{0}}(I)$. In that case, $\varphi(t) \in E_{\varphi_{0}}(I)$.

Proof. The operator $\varphi \mapsto \lambda \varphi-F(\varphi)$ is a local diffeomorphism of a neighborhood of $\psi(t)$ in $\mathcal{Y}_{k+1}$ into a neighborhood of $\lambda \psi-F(\psi)$ in $\mathcal{Y}_{k}$. By Theorem 5.1, the right side of (5.5) is a curve in $C\left(I, \mathcal{Y}_{k}\right)$ that has value $-\varphi_{1}$ at $t=0$. By Corollary 5.4, we may solve the equation uniquely for $\varphi(t)$ in a $\mathcal{Y}_{k+1}$-neighborhood of $\varphi_{0}$ and obtain that $\varphi(t) \in C\left(I, \mathcal{Y}_{k+1}\right)$. This requires to choose $T$ sufficiently small but uniformly in $\psi \in E_{\varphi_{0}}(I)$.

Formal differentiation of the equation solved by $\varphi(t)$ yields that

$$
\left(\lambda-L_{\varphi(t)}\right) \partial_{t} \varphi=\lambda b-\partial_{t} b=\left(\lambda-L_{\psi(t)}\right) b(t) .
$$

By the invertibility of the operator $\lambda-L_{\varphi(t)}$ and the known regularity of the right side, it follows that $\partial_{t} \varphi \in C\left(I, \mathcal{Y}_{k}\right)$ and has value $\varphi_{1}$ at $t=0$. Iterated differentiation yields that $\varphi(t) \in C_{(k+1,0)}(I ; \mathcal{Y})$ and has the desired coefficients in its Taylor series expansion up to order $k$. Moreover, the way the equation is solved, we have that

$$
\left\|\partial_{t}^{l} \varphi(t)-\varphi_{l}\right\|_{k+1-l} \leq R
$$

for $t \in I$. This completes the proof.

Proposition 5.6. For $\psi \in E_{\varphi_{0}}(I)$, let $\varphi(t) \in E_{\varphi_{0}}(I)$ be the solution curve given by the previous proposition. If $T$ is sufficiently small, the mapping

$$
\begin{gathered}
E_{\varphi_{0}}(I) \longrightarrow E_{\varphi_{0}}(I) \\
\psi(t) \longrightarrow \varphi(t)
\end{gathered}
$$

is a contraction in the metric induced by the norm $\|w\|_{1}=\sup _{t \in I}\|w(t)\|_{1}$, relative to which, $E_{\varphi_{0}}(I)$ is complete.

Proof. Given a curve $b(t)$ in $\mathcal{Y}_{q}$, we define a norm by $\|b\|_{q}=\sup _{t \in I}\|b(t)\|_{q}$. We shall only make use of the 1 and 0 norm, respectively.

Let $\psi_{1}$ and $\psi_{2}$ be two elements of $E_{\varphi_{0}}(I)$ and let $b_{1}$ and $b_{2}$ be the solutions to the corresponding approximate linearized equations with the same initial condition $\varphi_{1}$. We then have that

$$
b_{1}(t)=p_{\psi_{1}}(t, 0) \varphi_{1}, \quad b_{2}(t)=p_{\psi_{2}}(t, 0) \varphi_{1},
$$

where $p_{\psi_{1}}(t, s)$ and $p_{\psi_{2}}(t, 0)$ are the evolution operators of the linear equations $\partial_{t} v=L_{\psi_{1}(t)} v$ and $\partial_{t} v=L_{\psi_{2}(t)} v$, respectively. Consequently,

$$
b_{2}(t)-b_{1}(t)=\left(p_{\psi_{2}}(t, 0)-p_{\psi_{1}}(t, 0)\right) \varphi_{1},
$$

and using the identity

$$
p_{\psi_{2}}(t, 0) \varphi-p_{\psi_{1}}(t, 0) \varphi=-\int_{0}^{t} p_{\psi_{2}}(t, \tau)\left(L_{\psi_{2}(\tau)}-L_{\psi_{1}(\tau)}\right) p_{\psi_{1}}(\tau, 0) \varphi d \tau,
$$


we obtain the estimate

$$
\left\|b_{2}(t)-b_{1}(t)\right\|_{0} \leq C\left\|\varphi_{1}\right\|_{k} \int_{0}^{t}\left\|L_{\psi_{2}(\tau)}-L_{\psi_{1}(\tau)}\right\|_{k, 0} d \tau
$$

for some constant $C$. But we have observed that $\left\|L_{\psi_{2}(\tau)}-L_{\psi_{1}(\tau)}\right\|_{k, 0}$ is bounded by a constant times $\left\|\psi_{2}(\tau)-\psi_{1}(\tau)\right\|_{1}$. For small enough $R$, this last constant can be chosen uniformly. We then obtain that

$$
\left\|b_{2}-b_{1}\right\|_{0} \leq C T\left\|\varphi_{1}\right\|_{k}\left\|\psi_{2}-\psi_{1}\right\|_{1},
$$

showing that the map

$$
\psi(t) \mapsto b(t)
$$

is a contraction from the 1-norm to the 0-norm, with contraction factor arbitrarily small with $T$.

That the map $\psi(t) \mapsto \varphi(t)$ is a contraction now follows because the map $b(t) \mapsto \varphi(t)$ is uniformly $C^{1}$ from the 0 -norm to the 1-norm. This last map is simply the inverse of $\varphi \mapsto \lambda \varphi-F(\varphi)$ from $\mathcal{Y}_{1}$ to $\mathcal{Y}_{0}$, and we have that $\lambda-L_{\varphi(t)}$ is an isomorphism from $\mathcal{Y}_{1}$ to $\mathcal{Y}_{0}$, uniformly in $\psi(t)$ when $\psi(t)$ is close to $\varphi_{0}$.

In view of the previous results, there exists a unique fixed point $\varphi(t)$ of the map (5.6). Since $b(t)$ solves (4.5) with initial data $\varphi_{1}$, differentiating with respect to $t$ in (5.5) we obtain:

$$
\left(\lambda-L_{\varphi(t)}\right) \partial_{t} \varphi(t)=-\dot{b}(t)+\lambda b(t)=\left(\lambda-L_{\varphi(t)}\right) b(t),
$$

and since $\lambda-L_{\varphi(t)}$ is injective, we must have that

$$
b(t)=\partial_{t} \varphi(t) .
$$

We may now use this fact in carrying the time integral in (5.5), and conclude that

$$
\frac{d}{d t} \varphi(t)=F(\varphi(t))
$$

Thus, the fixed point $\varphi(t) \in E_{\varphi_{0}}(I)$ is a solution to the initial value problem (3.7).

We thus arrive at the following:

Theorem 5.7. Let $(M, J, \Omega)$ be a polarized Kähler manifold and let $G$ be a maximal compact subgroup of Aut $(M, J)$. The extremal flow equation

$$
\partial_{t} \omega_{t}=-\rho_{t}+\Pi_{t} \rho_{t}
$$

in $\mathfrak{M}_{\Omega, G}$ with a given initial data has a unique solution for a short time.

In fact, our proof carefully analyses how the time of existence depends upon the coefficients of the metric and its curvature tensor. Indeed, it shows that the local time of existence depends on the $L^{\infty}$-norm of the coefficients of the initial metric and its curvature operator. We can improve a bit the statement above in relation to the lifespan of the extremal flow. 
Corollary 5.8. Given an initial condition $\omega \in \mathfrak{M}_{\Omega, G}$, the extremal evolution equation has a unique solution on a maximal time interval $0 \leq t<T \leq \infty$. If $T<\infty$, then the maximum of the point wise norm of the curvature tensor blows up as $t \rightarrow T$.

The above blow up, if any, occurs on the Ricci part of the curvature tensor, rather than the full curvature tensor itself.

\section{Further remarks}

It is of course important to know when the extremal flow has solutions for all times. Indeed, once the local time existence is known, the next problem to consider is the use of the flow to show the existence of extremal metrics representing a given cohomology class $\Omega$, task that could be accomplished if we manage to prove global time existence and convergence of the metrics as $t \rightarrow \infty$.

This scheme could not possible work in all cases, as we already know of examples of polarized Kähler manifolds without extremal metrics [2]. But as a testing ground of its usefulness, we have started its analysis when pursuing extremal metrics on polarized manifolds $(M, J, \Omega)$ with $c_{1}<0$, or on polarized complex surfaces with $c_{1}>0$. The partial results obtained so far are quite encouraging.

We have two types of fairly strong reasons supporting our belief that this approach will produce extremal metrics in the said cases. The first of these is directly related to the flow itself, while the other one involves some relation between this flow and the study of families of extremal problems as we vary the cohomology class $\Omega$. We discuss them briefly in this section.

The evolution equation (3.6) implies evolution equations for various metric tensors associated to the varying metrics. For instance, the Ricci form evolves according to the equation

$$
\frac{d}{d t} \rho=-\frac{1}{2} \Delta \rho+\frac{i \partial \bar{\partial}(\pi s)}{2},
$$

the scalar curvature evolves according to the equation

$$
\frac{d}{d t} s=-\frac{1}{2} \Delta(s-\pi s)-2(\rho, i \partial \bar{\partial} G(s-\pi s)),
$$

and the Ricci potential evolves according to the equation

$$
\frac{d}{d t} \psi=-\frac{1}{2} \Delta \psi-2 G\left(\rho_{H}, i \partial \bar{\partial}(\psi+G(\pi s))\right)-\left(\pi s-s_{0}\right)+\frac{1}{2 \mu(M)} \int \psi(s-\pi s) d \mu .
$$

Here $\rho_{H}$ is the harmonic component of $\rho$, and $\mu(M)$ is the volume of $M$ relative to $\omega$.

The first of the equations above shows that the form $\rho$ is a solution to the heat equation for the time dependent Hodge Laplacian. One might expect that Hamilton's maximum principle (Theorem 9.1 in [11]) for solutions to the heat equation 
of the rough Laplacian could be extended to this new setting. If so, such a result would allow us to conclude that if the initial condition for $\rho$ has a sign, then that sign should be preserve along the flow (3.6) for $0 \leq t \leq T, T$ the lifespan of the solution. At the very least, such a result should hold for generic manifolds $(M, J)$.

We could then apply this to manifolds with no non-trivial holomorphic vector fields, such as any complex manifold $(M, J)$ with negative first Chern class, or most complex surfaces with positive first Chern classes. Notice that for the blow up of $\mathbb{C P}^{2}$ at one point, a manifold that carries non-trivial holomorphic vector fields, the positivity of the Ricci form is preserved along the flow. This makes it even more likely that such a result would also hold on any complex surface with positive $c_{1}$.

We may also refine our earlier Theorem 3.3 when dealing with a complex surface of positive first Chern class. Indeed, we have the following result, whose proof will be given elsewhere.

Theorem 6.1. Let $(M, J, \Omega)$ be a polarized complex surface of positive first Chern class. Given any Kähler metric $g$ in $\mathfrak{M}_{\Omega, G}$, the image of the holomorphy potential $\pi_{g} s_{g}$ is an interval contained in the set of positive real numbers, interval that only depends on $\Omega$ and not on $g$.

Thus, if for a given initial condition with positive Ricci curvature on a $c_{1}$ positive surface we have that solutions to the flow (3.6) exists for all time, and converge to an extremal metric as time goes to infinity, the extremal metric so obtained would have positive scalar curvature, as expected.

The preservation of the sign of the Ricci tensor should have very strong implications on the global analysis of (3.6). This property has been of utmost importance already in the work of Hamilton [11], and should remain so in the general analysis of our flow equation as well. If $c_{1}>0$, we could combine this with plausible global time existence results, and pass to a Cheeger-Gromov-Hausdorff limit, an important step towards settling the convergence issue.

We venture the following two conjectures.

Conjecture 6.2. Let $(M, J)$ be a complex manifold of Kähler type polarized by a Kähler class $\Omega$. If $c_{1}(M, J)<0$, there exists an initial condition to the extremal flow (3.7) equation so that the solution exists on $[0, \infty)$ and, as $t \rightarrow \infty$, converges to a metric of constant negative scalar curvature representing $\Omega$.

Conjecture 6.3. Let $(M, J)$ be a complex surface of positive first Chern class polarized by a Kähler class $\Omega$. Then there exists an initial condition to the extremal flow (3.7) equation so that the solution exists on $[0, \infty)$ and, as $t \rightarrow \infty$, converges to an extremal metric of positive scalar curvature representing $\Omega$.

The initial condition we have in mind in these two cases is given by a metric whose Ricci form is negative or positive, respectively. After the work of Yau [23] on the Calabi conjecture, we know we can always find this type of metrics on any given polarization.

These conjectures are further supported by the results in [21], that we proceed to describe in brief detail. For a complex manifold $(M, J)$ of complex dimension 
$n$, we denote by $\mathfrak{M}$ the space of Kähler metrics on $(M, J)$. As before, given a positive class $\Omega \in H^{1,1}(M, \mathbb{C}) \cap H^{2}(M, \mathbb{R})$, we let $\mathfrak{M}_{\Omega}$ be the space of of Kähler metrics whose Kähler forms represent $\Omega$. We shall also consider the space $\mathfrak{M}_{1}$ of Kähler metrics of volume one, and $\mathcal{K}_{1}$, the space of cohomology classes that can be represented by Kähler forms of metrics in $\mathfrak{M}_{1}$ :

$$
\mathcal{K}_{1}=\left\{\Omega \in H^{1,1}(M, \mathbb{C}): \Omega=[\omega] \text { for some } \omega \in \mathfrak{M}_{1}\right\} .
$$

Extremal metrics in $\mathfrak{M}_{\Omega}$ achieve the infimum of the functional $\Phi_{\Omega}$ in (2.4), and we have the lower bound (2.8):

$$
E(\Omega)=\int\left(\pi_{g} s_{g}\right)^{2} d \mu_{g}
$$

One approach to providing $(M, J)$ with a canonical shape would be to find critical points of the functional

$$
\begin{aligned}
\mathfrak{M}_{1} & \rightarrow \mathbb{R} \\
\omega & \mapsto \int_{M} s_{\omega}^{2} d \mu_{\omega} .
\end{aligned}
$$

A special metric $\omega$ of this type must have the following properties:

a) $\omega$ achieves the lower bound $E([\omega])$, that is to say, $\omega$ is extremal relative to the polarization defined by the Kähler class $\Omega=[\omega]$ that it represents;

b) the Kähler class $\Omega=[\omega]$ is a critical point of $E(\Omega)$ as a functional defined over $\mathcal{K}_{1}$.

Thus, the search for critical points of (6.2) —or strongly extremal metrics [18] achieving an optimal lower bound involves the solution of back-to-back minimization problems: the first solving for critical points of (2.4) within a fixed cohomology class $\Omega$, and the second solving for those classes that minimize the critical value $E(\Omega)$ as the class $\Omega$ varies within $\mathcal{K}_{1}$. Naturally, we separate the two problems by, in addition to (2.4), introducing the functional

$$
\begin{aligned}
\mathcal{K}_{1} & \rightarrow \mathbb{R} \\
\Omega & \mapsto E(\Omega)=\int_{M}(\pi s)^{2} d \mu,
\end{aligned}
$$

where the geometric quantities in the right are those associated with any $G$-invariant metric that represents $\Omega$, for $G$ a fixed maximal compact subgroup of the automorphism group of $(M, J)$. Its extremal points will be called either critical or canonical classes. We then have [20] the following:

Theorem 6.4. Let $\Omega$ be a cohomology class that is represented by a Kähler metric $g$, assumed to be invariant under the maximal compact subgroup $G$ of the biholomorphism group of $(M, J)$. Then $\Omega$ is critical class if and only if

$$
\int_{M}\left(\pi_{g} s_{g}\right)\left(\Pi_{g} \rho, \alpha\right) d \mu_{g}=0
$$


for any trace-free harmonic $(1,1)$-form $\alpha$. In this expression, $\rho$ is the Ricci form of the metric $g, \pi$ is the $L^{2}$ projection (3.2) onto the space of holomorphy potentials, and $\Pi$ is its lift (3.3) at the level of $(1,1)$-forms.

This theorem states that $\Omega$ is a critical class of (6.3) if and only if

$$
\int_{M}\left(\pi_{g} s_{g}\right)\left(\Pi_{g} \rho, \alpha\right) d \mu_{g}=0
$$

for any trace-free harmonic $(1,1)$-form $\alpha$. In other words, the form $\pi s \Pi \rho$ is $L^{2}$ perpendicular to the space of trace-free harmonic $(1,1)$-forms, and therefore, by Hodge decomposition, the class must be such that

$$
\pi s \Pi \rho=\lambda \omega+\partial G_{\partial}\left(\partial^{*}(\pi s \Pi \rho)\right)+\partial^{*} G_{\partial}(\partial(\pi s \Pi \rho)),
$$

for $\lambda$ equal to the $L^{2}$-projection of $(\pi s)^{2}$ onto the constants, divided by $2 n$ :

$$
\lambda=\frac{1}{2 n} \int(\pi s)^{2} d \mu_{g} .
$$

In order to study the existence of critical classes, we may consider [21] the evolution equation

$$
\frac{d \Omega}{d t}=\pi s \Pi \rho-\lambda \omega+\partial G_{\partial}\left(\partial^{*}(\pi s \Pi \rho)\right)+\partial^{*} G_{\partial}(\partial(\pi s \Pi \rho)) .
$$

The flow equation (6.6) defines a dynamical system on $\mathcal{K}_{1}$ provided the solutions remain in $\mathcal{K}_{1}$ throughout time. Unfortunately, this is not true in general [21].

In the generic case where all non-trivial holomorphic vector fields of $(M, J)$ have no zeroes, equation (6.6) can be extended to a dynamical system on

$$
\overline{\mathcal{K}}_{1}=\left\{\Omega \in H^{1,1}(M, \mathbb{C}) \cap H^{2}(M, \mathbb{R}): \frac{\Omega^{n}}{n !}=1\right\} .
$$

Indeed, given $\Omega \in \overline{\mathcal{K}}_{1}$, let us define the function

$$
s_{\Omega}:=4 \pi n \frac{c_{1} \cdot \Omega^{n-1}}{\Omega^{n}} .
$$

If $\Omega$ were a Kähler class represented by a metric $g$, this function would be precisely the holomorphy potential $\pi_{g} s_{g}$. The equation

$$
\frac{d}{d t} \Omega=2 \pi s_{\Omega} c_{1}-\frac{s_{\Omega}^{2}}{2 n} \Omega
$$

extends (6.6), which as such is defined only on $\mathcal{K}_{1}$, all the way to a dynamical system on $\overline{\mathcal{K}}_{1}$.

Solutions to (6.7) with initial data in $\overline{\mathcal{K}}_{1}$ remain in $\overline{\mathcal{K}}_{1}$. In fact, we have that [21] 
Theorem 6.5. Suppose that all non-trivial holomorphic vector fields of $(M, J)$ have no zeroes. Then solutions to (6.7) with initial data in $\overline{\mathcal{K}}_{1}$ converge, as $t \rightarrow \infty$, to a stationary point of the equation in the space $\overline{\mathcal{K}}_{1}$.

It is then of natural interest to see if solutions to the equation with Cauchy data given by a positive class, that is to say, an element of $\mathcal{K}_{1}$, remain positive thereafter. We already know [21] of examples where this is not so, with solutions to the flow equation that are initially in the Kähler cone but that, in converging to a critical point of the flow in $\overline{\mathcal{K}}_{1}$, must eventually leave the cone through its walls.

In fact, this situation occurs already on complex surfaces, where the stability of $\mathcal{K}_{1}$ under the flow (6.7) can be analyzed using a criterion giving necessary and sufficient for a cohomology class to be Kähler, criterion that extends that of Nakai for integral classes. Applied to our problem, if the Chern number $c_{1}^{2} \neq 0$, we have that a path $\Omega_{t}$ solving (6.7) with initial condition in $\mathcal{K}_{1}$ stays there forever after if, and only if,

$$
\Omega_{0} \cdot[D]+8 \pi^{2}\left(c_{1} \cdot \Omega_{0}\right)\left(c_{1} \cdot[D]\right)\left(\frac{e^{c_{1}^{2} t}-1}{c_{1}^{2}}\right)>0
$$

for all $t \geq 0$ and for all effective divisors $D$ in $(M, J)$. When $c_{1}^{2}=0$ we still obtain a similar criterion, replacing the expression in parentheses above by its limit $t$ as $c_{1}^{2} \rightarrow 0$.

This forward stability of the Kähler cone holds in very general situations, as can be seen by a run-down of the various cases in the Enriques-Kodaira classification of complex surfaces [21]. In particular, it holds if the complex surface has a signed first Chern class $c_{1}$, condition under which all solutions to the flow (6.7) that start in $\mathcal{K}_{1}$ stay there forever after, and as $t \rightarrow \infty$, they either converge to the only critical class $\sqrt{2}\left(\operatorname{sgn} c_{1}\right) c_{1} / c_{1}^{2}$ of (6.3) if $c_{1}^{2}>0$, or all classes are critical and the flow is constant if $c_{1}=0$.

Notice that the positivity condition above involves the evaluation of $c_{1}$ over the divisor $D$, and only in the case when there are effective divisors $D$ for which $c_{1} \cdot[D]$ changes sign from one to another could the condition fail to hold. Merely fixing the sign of $c_{1}$ prevents this from happening, but the counterpart to that is of great interest. It shows that the existence of divisors on which $c_{1}$ achieves values of opposite signs is in effect part of the reason why the the Kähler cone might be poorly behaved in relation to the flow (6.6).

When the surface in question has positive first Chern class and carries nontrivial holomorphic fields, the forward stability of the Kähler cone under the flow (6.6) seems to hold also, though we have only verified that for the case of $\mathbb{C P}^{2}$ blown up at one point.

In higher dimension and for manifolds $(M, J)$ where $c_{1}$ is either positive or negative, the space of Kähler classes is also forward stable under the flow (6.7). As a matter of fact, there is a positivity criterion that generalizes the one outlined above for surfaces, which guarantees forward stability of the Kähler cone under the flow. Manifolds with signed first Chern classes meet this criterion, though for these 
particular cases one can also give a direct argument that proves the flow stability of the cone.

All of these facts combined give further support to the conjectures made earlier. We end up venturing a final one.

Conjecture 6.6. Suppose the flow equation (6.7) with initial data in the Kähler cone converges to a stationary point that is outside it. Then the extremal Kähler cone is not a closed subset of the Kähler cone.

In other words, under the given hypothesis, there should exist cohomology classes in the Kähler cone that cannot be represented by extremal metrics.

\section{References}

[1] M. Atiyah, Convexity and commuting Hamiltonians, Bull. London Math. Soc. 14 (1982), $1-15$.

[2] D. Burns and P. DE BARTolomeis, Stability of vector bundles and extremal metrics, Invent. Math. 92 (1988), 403-407.

[3] E. CAlabi, Extremal Kähler metrics, In: "Seminars on Differential Geometry", S. T. Yau (ed.), Annals of Mathematics Studies, Princeton University Press, 1982, 259-290.

[4] E. CALABI, "Extremal Kähler Metrics II", In: "Differential Geometry and Complex Analysis", Chavel \& Farkas (eds.), Springer-Verlag, 1985, 95-114.

[5] H.-D. CAO, Deformation of Kähler metrics to Kähler-Einstein metrics on compact Kähler manifolds, Invent. Math. 81 (1985), 359-372.

[6] J. Eells and J. H. SAMPSON, Harmonic mappings of Riemannian manifolds, Amer. J. Math. 86 (1964), 109-160.

[7] A. FUTAKI, "Kähler-Einstein metrics and integral invariants", Lect. Notes in Math. 1314, Springer-Verlag, 1987.

[8] A. FUTAKI and T. MABUCHI, Bilinear forms and extremal Kähler vector fields associated with Kähler classes, Math. Ann. 301 (1995), 199-210.

[9] V. Guillemin and S. STERnBERG, Convexity properties of the moment mapping, Invent. Math. 67 (1982), 491-513.

[10] R. Hamilton, The Ricci flow on surfaces, Contemp. Math., 71 (1988), 237-262.

[11] R. Hamilton, Three manifolds with positive Ricci curvature, J. Differential Geom. 17 (1982), 255-306.

[12] T. KATO, "Abstract differential equations and non-linear mixed problems", Lezioni Fermiane, Scuola Norm. Sup. Pisa Cl. Sci., 1985.

[13] C. LeBrun and S. R. SimancA, On Kähler Surfaces of Constant Positive Scalar Curvature, J. Geom. Anal. 5 (1995), 115-127.

[14] C. LeBrun and S. R. SimancA, Extremal Kähler Metrics and Complex Deformation Theory, Geom. Func. Anal. 4 (1994), 298-336.

[15] C. LeBRUn and S. R. SimAnCA, On the Kähler Classes of Extremal Metrics, In: "Geometry and Global Analysis", First MSJ Intern. Res. Inst. Sendai, Japan, Kotake, Nishikawa and Schoen (eds.), 1993.

[16] M. LEVINE, A remark on extremal Kähler metrics, J. Differential Geom. 21 (1986), 73-77.

[17] S. R. SimancA, A K-energy characterization of extremal Kähler metrics, Proc. Amer. Math. Soc. 128 (2000), 1531-1535.

[18] S. R. SimancA, Strongly extremal Kähler metrics, Ann. Global Anal. Geom. 18 (2000), $29-46$.

[19] S. R. Simanca, Precompactness of the Calabi Energy, Internat. J. Math. 7 (1996), 245254. 
[20] S. R. Simanca and L. Stelling, Canonical Kähler classes, Asian J. Math. 5 (2001), 585-598.

[21] S. R. SimAnCA and L. Stelling, The dynamics of the energy of a Kähler class, Commun. Math. Phys. 255 (2005), 363-389.

[22] G. Tian, Kähler-Einstein metrics with positive scalar curvature, Invent. Math. 130 (1997), $1-37$.

[23] S. T. YAU, On the Ricci curvature of a compact Kähler manifold and the complex MongeAmpère equation I, Comm. Pure. Applied Math. 31 (1978), 339-411.

Institute for Mathematical Sciences

Stony Brook, NY 11794

santiago@math.sunysb.edu 